SELECCIONES MATEMÁTICAS
Universidad Nacional de Trujillo
ISSN: 2411-1783 (Online)
Vol. $05(02): 121-136(2018)$

\title{
Local Well-posedness of a Nutku-Og̃uz-Burgers System With Time Dependent Coefficients
}

\section{Buena formulación local de un sistema de Nutku-Õ̃uz-Burgers con coeficientes dependientes del tiempo}

Juan Montealegre* ${ }_{\text {and }}$ Gladys Cruz ${ }^{\dagger}$

Received, May. 06, 2018

Accepted, Set. 15, 2018

DOI: http://dx.doi.org/10.17268/sel.mat.2018.02.01

\begin{abstract}
In this paper we study the local well-posedness of the initial value problem for a Nutku-Og̃u-Burgers system with time dependent coefficients, formed by two Korteweg-de Vries equations coupled through the non-linear terms. The system appears as a model of wave propagation in a shallow channel with variable bottom surface, in which both nonlinear and dispersive effects are relevant. The proof of existence and uniqueness of local solution and the continuous dependence on the initial data of the local solution in Sobolev spaces $H^{s}(\mathbb{R}) \times H^{s}(\mathbb{R}), s>3 / 2$, are based on the works [9] and [17].
\end{abstract}

Keywords. Initial alue problem, Korteweg-de Vries equations, Local well-posedness, Sobolev spaces.

\section{Resumen}

En este artículo estudiamos la buena formulación local de valor inicial para un sistema Nutku-Oz̃uz-Burgers con coeficientes dependientes del tiempo, formado por dos ecuaciones de Korteweg-de Vries acopladas a través de los términos no lineales. El sistema aparece como un modelo de la propagación de ondas en un canal de poca profundidad con la superficie del fondo variable, en el cual tanto los efectos no lineales como los dispersivos son relevantes. Las demostraciones de la existencia y unicidad de la solución local y la dependencia continua de la solución local respecto de los datos iniciales en los espacios de Sobolev $H^{s}(\mathbb{R}) \times H^{s}(\mathbb{R}), s>3 / 2$, se basan en los trabajos [9] y [17].

Palabras clave. Problema de valor inicial, Ecuación de Korteweg-de Vries, Buena formulación local, Espacios de Sobolev.

1. Introduction. In this paper we analyze the local well-posedness of the initial-value problem associated with the system

$$
\left\{\begin{array}{l}
\partial_{t} u+\partial_{x}^{3} u+\alpha(t) u^{p} \partial_{x} u+v^{p} \partial_{x} v+\partial_{x}\left(u v^{p}\right)-\mu \partial_{x}^{2} u=0 \\
\partial_{t} v+\partial_{x}^{3} v+u^{p} \partial_{x} u+\beta(t) v^{p} \partial_{x} v+\partial_{x}\left(u^{p} v\right)-\mu \partial_{x}^{2} v=0 \\
u(x, 0)=\varphi(x) \\
v(x, 0)=\psi(x)
\end{array}\right.
$$

where $u=u(x, t)$ and $v=v(x, t)$ are real value functions, $(x, t) \in \mathbb{R} \times[0,+\infty[, \varphi$ and $\psi$ are initial data, $\alpha(t)$ and $\beta(t)$ are real non-negative, continuous and bounded functions $\alpha(t)+\beta(t)=1$ and the exponent $p$ is an integer greater than or equal to one.

\footnotetext{
*Pontificia Universidad Católica del Perú, Departamento de Ciencias. Av. Universitaria 1801, San Miguel-Lima 32, ú (jmscott@pucp.edu.pe).

†Universidad Nacional Tecnológica de Lima Sur, Facultad de Ingeniería y Gestión. Cruce de Av. Central y Av. Bolivar, Villa el SalvadorLima 42, Perú (gladyscy60@gmail.com)
} 
The system in (1.1) is a generalized version of the system

$$
\left\{\begin{array}{l}
\partial_{t} u+\partial_{x}^{3} u+\alpha u \partial_{x} u+v \partial_{x} v+\partial_{x}(u v)=0 \\
\partial_{t} v+\partial_{x}^{3} v+u \partial_{x} u+\beta v \partial_{x} v+\partial_{x}(u v)=0
\end{array}\right.
$$

where $\alpha, \beta$ are real positive constants such that $\alpha+\beta=1$ stablished by Y. Nutku y Ö. Õ̃uz [18] studied by J. Montealegre [15, 16]. The system (1.2), which has the structure of two Korteweg-de Vries equations coupled by the non-linear terms, it appears as a propagation model of waves in physical systems in which both nonlinear effects and the dispersives are relevant (see, for example, [4, 5, 14]). Different properties of the system (1.2) have been studied, including the theory of local well-posedness in spaces of high regularity [15] and its extension to spaces of low regularity [16].

Our purpose in this paper is to show the local well-posedness of the problem (1.1) in $\mathbb{H}^{s}=H^{s} \times H^{s}$ with $s>\frac{3}{2}$, that is, we will prove that there is $T>0$ and $\vec{u}: \mathbb{R} \times\left[0, T\left[\rightarrow \mathbb{R}^{2}, \vec{u}=(u, v)\right.\right.$ is the unique solution of the initial value problem (1.1) which depends continuously on the initial data whenever $\vec{\varphi}=(\varphi, \psi) \in \mathbb{H}^{s}$.

We shall use standard notation: we consider an interval $I \subseteq \mathbb{R}$, by $L^{\infty}(I)$ we shall denote the space of measurable essentially bounded functions in $I$ with its usual norm. The Fourier transform of $u$ is defined as $\widehat{u}(\xi)=$ $(2 \pi)^{-1 / 2} \int_{\mathbb{R}} e^{-i \xi x} f(x) d x$, and the inverse Fourier transform of $\check{u}$ is given by $\check{u}(x)=(2 \pi)^{-1 / 2} \int_{\mathbb{R}} e^{i \xi x} f(\xi) d \xi$. For each $s \in \mathbb{R}$, we shall denote by $H^{s}=H^{s}(\mathbb{R})$ the Sobolev space of order $s$ defined as the completion of the Schwartz space $\mathcal{S}=\mathcal{S}(\mathbb{R})$ with respect to the norm

$$
\|u\|_{H^{s}}^{2}=\int_{\mathbb{R}}\left(1+\xi^{2}\right)^{s}|\widehat{u}(\xi)|^{2} d \xi
$$

and we denote by $\mathbb{H}^{s}=H^{s} \times H^{s}$ with the norm $\|\vec{u}\|_{\mathbb{H}^{s}}^{2}=\left\|u_{1}\right\|_{H^{s}}^{2}+\left\|u_{2}\right\|_{H^{s}}^{2}$ for $\vec{u}=\left(u_{1}, u_{2}\right)$.

If $X$ is an arbitrary Banach space and $T$ is a positive real number, we denoted by $L^{\infty}([0, T]: X)$ the Banach space of measurable functions $u:[0, T] \rightarrow X$ such that $t \mapsto\|u(t)\|_{X}$ belongs to $L^{\infty}([0, T])$ and $C([0, T]: X)$ the vector subspace of $L^{\infty}([0, T]: X)$ of all continuous functions endowed with their usual norm. We denote by $J^{s}=\left(I-\partial_{x}^{2}\right)^{\frac{s}{2}}$ and $D^{s}=\left(-\partial_{x}^{2}\right)^{\frac{s}{2}}$, the Bessel and Riesz potentials of order $-s$ respectively. Let $A$ and $B$ two operators, then the commutator is given by $[A, B]=A B-B A$. Thus, $\left[J^{s}, u\right] v=J^{s}(u v)-u J^{s} v$ in which $u$ is regarded as a multiplication operator.

The following lemmas will be useful in the next sections.

Lemma 1.1. Let $u, v \in \mathcal{S}\left(\mathbb{R}^{n}\right)$ be two real functions, $s>1+\frac{n}{2}$ and $t \geq 1$. Then, there exists $C=$ $C(s, t, n)>0$ such that

$$
\left|\left\langle u, v \partial^{\alpha} u\right\rangle_{H^{t}}\right| \leq C\left[\|\nabla v\|_{H^{s-1}}\left\|u^{2}\right\|_{H^{t}}+\|\nabla v\|_{H^{t-1}}\|u\|_{H^{s}}\|u\|_{H^{t}}\right]
$$

where $|\alpha|=1$.

Proof. See [11, Lemma A.5.]

Lemma 1.2. Let $u, v \in \mathcal{S}(\mathbb{R}), 1<p<\infty$ and $s>0$, then

$$
\left\|J^{s}(u v)\right\|_{L^{p}} \leq C\left(\|u\|_{L^{p_{1}}}\left\|J^{s} v\right\|_{L^{p_{2}}}+\left\|J^{s} u\right\|_{L^{p_{3}}}\|v\|_{L^{p_{4}}}\right)
$$

and

$$
\left\|\left[J^{s}, u\right] v\right\|_{L^{p}} \leq C\left(\left\|\partial_{x} u\right\|_{L^{p_{1}}}\left\|J^{s-1} v\right\|_{L^{p_{2}}}+\left\|J^{s} u\right\|_{L^{p_{3}}}\|v\|_{L^{p_{4}}}\right)
$$

where $\left.p_{2}, p_{3} \in\right] 1, \infty\left[\right.$ and $p_{1}, p_{4}$ are such that $\frac{1}{p_{1}}+\frac{1}{p_{2}}=\frac{1}{p}=\frac{1}{p_{3}}+\frac{1}{p_{4}}$.

Proof. See [13]

The inequality (1.5) is also true if $p_{1}=p_{4}=\infty$ and $p_{2}=p_{3}=p$ as shown in [13, Lema X1].

2. The linear problem. Consider the linear problem associated with (1.1), which in its vector form is written

$$
\left\{\begin{array}{l}
\partial_{t} \vec{u}(t)+A_{\mu} \vec{u}(t)=0, x \in \mathbb{R}, t>0 \\
\vec{u}(0)=\vec{\phi}
\end{array}\right.
$$

where $\vec{u}=(u, v), \vec{\phi}=(\varphi, \psi)$ and $A_{\mu}$ is the operator defined by

$$
\left\{\begin{array}{l}
\mathcal{D}\left(A_{\mu}\right)=\mathbb{H}^{s+3}, s \geq 0 \\
A_{\mu} \vec{u}=\left(\partial_{x}^{3} u-\mu \partial_{x}^{2} u, \partial_{x}^{3} v-\mu \partial_{x}^{2} v\right), \vec{u}=(u, v) \in \mathbb{H}^{s+3} .
\end{array}\right.
$$


Proposition 2.1. The operator $-A_{\mu}: \mathbb{H}^{s+3} \subseteq \mathbb{H}^{s} \rightarrow \mathbb{H}^{s}$ is m-dissipative in $\mathbb{H}^{s}, s \geq 0$.

Proof. First we note that $-A_{\mu} \vec{u} \in \mathbb{H}^{s}$, since if $\vec{u}=(u, v) \in \mathbb{H}^{s+3}$, then the definition of $-A_{\mu}$, the properties of the norm and the inner product in $\mathbb{H}^{s}$, the inequality $\left\|\partial_{x}^{k} u\right\|_{H^{s}} \leq\|u\|_{H^{s+k}}$ and because $H^{s+3} \hookrightarrow H^{s+2}$ we get

$$
\left\|-A_{\mu} \vec{u}\right\|_{\mathbb{H}^{s}}^{2}=\left(1+\mu^{2}\right)\left[\left\|\partial_{x}^{2} u\right\|_{H^{s}}^{2}+\left\|\partial_{x}^{2} v\right\|_{H^{s}}^{2}\right]-2 \mu\left[\left\langle\partial_{x}^{3} u, \partial_{x}^{2} u\right\rangle_{H^{s}}+\left\langle\partial_{x}^{3} v, \partial_{x}^{2} v\right\rangle_{H^{s}}\right]
$$

Using integration by parts, in (2.3) we have

$$
\left\|-A_{\mu} \vec{u}\right\|_{\mathbb{H}^{s}}^{2} \leq\left(1+\mu^{2}\right)\|\vec{u}\|_{\mathbb{H}^{s+3}}^{2}
$$

Therefore $-A_{\mu} \vec{u} \in \mathbb{H}^{s}$.

The definition of $-A_{\mu}$, the properties of the inner product and integration by parts, we obtain

$$
\left\langle-A_{\mu} \vec{u}, \vec{u}\right\rangle_{\mathbb{H}^{s}}=-\mu\left(\left\|\partial_{x} u\right\|_{H^{s}}^{2}+\left\|\partial_{x} v\right\|_{H^{s}}^{2}\right) \leq 0 .
$$

Getting that operator $-A_{\mu}$ is negative, therefore $-A_{\mu}$ is dissipative in $\mathbb{H}^{s}$.

The operator $I+A_{\mu}$ is surjective, that is, for each $\vec{v}=(u, v) \in \mathbb{H}^{s}$ there exists $\vec{u}=(f, g) \in \mathbb{H}^{s+3}$ such that $\left(I+A_{\mu}\right) \vec{u}=\vec{v}$. In fact, let $\vec{v}=(u, v) \in \mathbb{H}^{s}$, then $\vec{u}=(f, g)$ with $\widehat{f}(\xi)=\frac{\widehat{u}(\xi)}{1+\mu \xi^{2}-i \xi^{3}}$ and $\widehat{g}(\xi)=\frac{\widehat{v}(\xi)}{1+\mu \xi^{2}-i \xi^{3}}$ satisfy $\left(I+A_{\mu}\right)(f, g)=(u, v)$. To conclude the proof it will be shown that $\vec{u} \in \mathbb{H}^{s+3}$. Indeed, we have

$$
\|\vec{u}\|_{\mathbb{H}^{s+3}}^{2}=\|f\|_{H^{s+3}}^{2}+\|g\|_{H^{s+3}}^{2}
$$

where

$$
\|f\|_{H^{s+3}}^{2}=\int_{\mathbb{R}}\left(1+\xi^{2}\right)^{s+3} \frac{|\widehat{u}(\xi)|^{2}}{\left(1+\mu \xi^{2}\right)^{2}+\xi^{6}} d \xi \leq C \int_{\mathbb{R}}\left(1+\xi^{2}\right)^{s+3} \frac{|\widehat{u}(\xi)|^{2}}{\left(1+\xi^{2}\right)^{3}} d \xi=C\|u\|_{H^{s}}^{2}
$$

for which the inequalities $\left(1+\mu \xi^{2}\right)^{2}+\xi^{6} \geq C\left(1+\xi^{2}\right)^{3}$ were used. In the same way,

$$
\|g\|_{H^{s+3}}^{2} \leq C\|v\|_{H^{s}}^{2} .
$$

Hence, in (2.4) we obtain

$$
\|\vec{u}\|_{\mathbb{H}^{s+3}}^{2} \leq C\left(\|u\|_{H^{s}}^{2}+\|v\|_{H^{s}}^{2}\right)=C\|\vec{v}\|_{\mathbb{H}^{s}}^{2}
$$

which implies that $\vec{u} \in \mathbb{H}^{s+3}$.

Theorem 2.2. If $\mu>0$, the $-A_{\mu}$ operator is the generator of a semigroup of contractions $\left\{\vec{W}_{\mu}(t)\right\}_{t \geq 0}$ in $\mathbb{H}^{s}$ with $s \geq 0$, such that for any $\vec{\phi} \in \mathbb{H}^{s}$ the function

$$
\vec{W}_{\mu}(\cdot) \vec{\phi}: \mathbb{R}_{0}^{+} \rightarrow \mathbb{H}^{s}
$$

is the unique solution of the initial value problem (2.1) in

$$
C\left(\left[0,+\infty\left[: \mathbb{H}^{s}\right) \cap C^{1}\left(\left[0+\infty\left[: \mathbb{H}^{s-2}\right) .\right.\right.\right.\right.
$$

Moreover, if $\vec{\phi}=(\varphi, \psi) \in \mathbb{H}^{s}$ then

$$
\vec{W}_{\mu}(t) \vec{\phi}=\left(W_{\mu}(t) \varphi, W_{\mu}(t) \psi\right)
$$

where $W_{\mu}(t)$ are the Fourier multipliers defined by

$$
\widehat{W_{\mu}(t)} \varphi(\xi)=e^{\left(i \xi^{3}-\mu \xi^{2}\right) t} \widehat{\varphi}(\xi),
$$

and for each $t \geq 0$ we have $\vec{W}_{\mu}(t) \in \mathcal{L}\left(\mathbb{H}^{s}: \mathbb{H}^{s+\sigma}\right)$ for any $\sigma \geq 0$ with

$$
\left\|\vec{W}_{\mu}(t) \vec{\phi}\right\|_{\mathbb{H}^{s+\sigma}} \leq K_{\sigma} \sqrt{1+\frac{1}{(2 \mu t)^{\sigma}}}\|\vec{\phi}\|_{\mathbb{H}^{s}} .
$$


Proof. The first and the second statements are consequences of proposition 2.1, the Lumer-Phillips's theorem and the theory of semigroups of linear operators. To obtain (2.5) and (2.6) it is sufficient to take the Fourier transform in the spatial variable, solve the resulting system of ordinary differential equations and use the function $\vec{W}_{\mu}(\cdot) \vec{\phi}$ which is the unique solution of the problem $(2.1)$.

Now we will prove the inequality (2.7). If $\vec{\phi}=\left(\varphi_{1}, \varphi_{2}\right) \in \mathbb{H}^{s}$ we have

$$
\left\|\vec{W}_{\mu}(t) \vec{\phi}\right\|_{\mathbb{H}^{s+\sigma}}^{2}=\sum_{j=1}^{2} \int_{\mathbb{R}}\left(1+\xi^{2}\right)^{s+\sigma}\left|e^{\left(i \xi^{3}-\mu \xi^{2}\right) t} \widehat{\varphi_{1}}(\xi)\right|^{2} d \xi
$$

Since $\left(1+\xi^{2}\right)^{\sigma} \leq C\left(1+\xi^{2 \sigma}\right)$ for $j=1,2$, we obtain that

$$
\left(1+\xi^{2}\right)^{s+\sigma}\left|e^{\left(i \xi^{3}-\mu \xi^{2}\right) t} \widehat{\varphi_{j}}(\xi)\right|^{2} \leq C\left(1+\xi^{2}\right)^{s} e^{-2 \mu \xi^{2} t}\left|\widehat{\varphi_{j}}(\xi)\right|^{2}+C \xi^{2 \sigma}\left(1+\xi^{2}\right)^{s} e^{-2 \mu \xi^{2} t}\left|\widehat{\varphi_{j}}(\xi)\right|^{2}
$$

Hence,

$$
\begin{aligned}
\left\|\vec{W}_{\mu}(t) \vec{\phi}\right\|_{\mathbb{H}^{s+\sigma}}^{2} \leq & C \int_{\mathbb{R}} e^{-2 \mu \xi^{2} t}\left(1+\xi^{2}\right)^{s}\left(\left|\widehat{\varphi_{1}}(\xi)\right|^{2}+\left|\widehat{\varphi_{2}}(\xi)\right|^{2}\right) d \xi \\
& +C \int_{\mathbb{R}} \xi^{2 \sigma} e^{-2 \mu \xi^{2} t}\left(1+\xi^{2}\right)^{s}\left(\left|\widehat{\varphi_{1}}(\xi)\right|^{2}+\left|\widehat{\varphi_{2}}(\xi)\right|^{2}\right) d \xi \\
\equiv & I_{1}+I_{2},
\end{aligned}
$$

where

$$
I_{1} \leq C \int_{\mathbb{R}}\left(1+\xi^{2}\right)^{s} \sup _{\xi \in \mathbb{R}} e^{-2 \mu \xi^{2} t}\left(\left|\widehat{\varphi_{1}}(\xi)\right|^{2}+\left|\widehat{\varphi_{2}}(\xi)\right|^{2}\right) d \xi=C \sup _{\xi \in \mathbb{R}} e^{-2 \mu \xi^{2} t}\|\vec{\phi}\|_{\mathbb{H}^{s}}^{2}
$$

and using the inequality $\xi^{2 \sigma} e^{-2 \xi^{2} \mu t} \leq \frac{\sigma^{\sigma} e^{-\sigma}}{(2 \mu t)^{\sigma}}$ valid for all $\mu>0, t \geq 0$ and $\sigma \geq 0$, we obtain

$$
I_{2} \leq \frac{C \sigma^{\sigma} e^{-\sigma}}{(2 \mu t)^{\sigma}}\|\vec{\phi}\|_{\mathbb{H}^{s}}^{2}
$$

Then in $(2.8)$ we get

$$
\left\|\vec{W}_{\mu}(t) \vec{\phi}\right\|_{\mathbb{H}^{s+\sigma}}^{2} \leq C \sup _{\xi \in \mathbb{R}} e^{-2 \mu \xi^{2} t}\|\vec{\phi}\|_{\mathbb{H}^{s}}^{2}+\frac{C \sigma^{\sigma} e^{-\sigma}}{(2 \mu t)^{\sigma}}\|\vec{\phi}\|_{\mathbb{H}^{s}}^{2} \leq K_{\sigma}^{2}\left(1+\frac{1}{(2 \mu t)^{\sigma}}\right)\|\vec{\phi}\|_{\mathbb{H}^{s}}^{2}
$$

where $K_{\sigma}^{2}=\max \left\{C \sup _{\xi \in \mathbb{R}} e^{-2 \mu \xi^{2} t}, C \sigma^{\sigma} e^{-\sigma}\right\}$, as we wanted to shown.

3. The integral equation associated with the initial value problem. The initial value problem (1.1) is written in vector form

$$
\left\{\begin{array}{l}
\partial_{t} \vec{u}+A_{\mu} \vec{u}+\partial_{x} F(\vec{u})=0 \\
\vec{u}(0)=\vec{\phi}
\end{array}\right.
$$

where $\vec{u}=(u, v), \vec{\phi}=(\varphi, \psi)$, the operator $A_{\mu}$ was defined in (2.2) and $F$ is the vector function defined by

$$
F(\vec{u})=\left(\frac{\alpha(t)}{p+1} u^{p+1}+\frac{1}{p+1} v^{p+1}+u v^{p}, \frac{1}{p+1} u^{p+1}+\frac{\beta(t)}{p+1} v^{p+1}+u^{p} v\right) .
$$

It is readily seen that if $\mu>0$ is fixed and $\vec{u}=(u, v)$ is solution of (3.1), then $\vec{u}$ is solution of the integral equation

$$
\vec{u}(t)=\vec{W}_{\mu}(t) \vec{\phi}-\int_{0}^{t} \vec{W}_{\mu}(t-\tau) \partial_{x} F(\vec{u}(\tau)) d \tau
$$

In this section we show the existence of a solution of the integral equation (3.3) that is solution of the problem (3.1). In the proof of the main result, theorem 3.2, we use Banach's fixed-point theorem to show the existence of solution of the integral equation and a classical argument of T. Kato and H. Fujita [12] to show the uniqueness of the solution. 
Suppose that $\vec{\phi} \in \mathbb{H}^{s}$ with $s>\frac{3}{2}$ and $\vec{\phi} \neq 0$. For $T \geq 0$ any, we define

$$
\mathcal{E}_{s}(T)=\left\{\vec{u} \in C\left([0, T]: \mathbb{H}^{s}\right):\left\|\vec{u}(t)-\vec{W}_{\mu}(t) \vec{\phi}\right\|_{\mathbb{H}^{s}} \leq\|\vec{\phi}\|_{\mathbb{H}^{s}}, 0<t \leq T\right\}
$$

with the metric

$$
d(\vec{u}, \vec{v})=\sup _{0 \leq t \leq T}\|\vec{u}(t)-\vec{v}(t)\|_{\mathbb{H}^{s}}, \quad \text { for } \vec{u}, \vec{v} \in \mathcal{E}_{s}(T)
$$

it is readily seen that $\left(\mathcal{E}_{s}(T), d\right)$ is a complete metric space. For $\vec{\phi} \in \mathbb{H}^{s}$ fixed, we define for any $\vec{u} \in \mathcal{E}_{s}(T)$ the application $\Theta$ by

$$
\Theta \vec{u}(t)=\vec{W}_{\mu}(t) \vec{\phi}-\int_{0}^{t} \vec{W}_{\mu}(t-\tau) \partial_{x} F(\vec{u}(\tau)) d \tau, t \in[0, T]
$$

In the follow proposition we study the properties of the application $\Theta$ defined on $\mathcal{E}_{s}(T)$.

Proposition 3.1. If $\alpha, \beta \in C(\mathbb{R}) \cap L^{\infty}(\mathbb{R}), \mu>0$ and $\vec{\phi} \in \mathbb{H}^{s}$ with $s>\frac{3}{2}$, then there exists $T_{\mu}=$ $T_{\mu}\left(\|\vec{\phi}\|_{\mathbb{H}^{s}}, \alpha, \beta, s, \mu\right) \in[0, T]$ such that $\Theta: \mathcal{E}_{s}\left(T_{\mu}\right) \rightarrow \mathcal{E}_{s}\left(T_{\mu}\right)$ is a contraction.

Proof. Let be given any $T>0, \Theta \vec{u}(t) \in \mathbb{H}^{s}$ for all $t \in[0, T]$. Indeed, if $\vec{\phi} \in \mathbb{H}^{s}$, by the theorem 2.2 it holds that $\vec{W}_{\mu}(t) \vec{\phi} \in \mathbb{H}^{s}$. On the other hand, since $H^{s}$ is a Banach algebra, it follows that $\partial_{x} F(\vec{u}(\cdot)) \in$ $C\left([0, T]: \mathbb{H}^{s-1}\right)$, then the integral $\int_{0}^{t} \vec{W}_{\mu}(t-\tau) \partial_{x} F(\vec{u}(\tau)) d \tau \in \mathbb{H}^{s-1}$. Moreover, choosing $\sigma=1$ in $(2.7)$ we obtain $\vec{W}_{\mu}(t-\cdot) \partial_{x} F(\vec{u}(\cdot)) \in C\left([0, T]: \mathbb{H}^{s}\right)$ and

$$
\left\|\int_{0}^{t} \vec{W}_{\mu}(t-\tau) \partial_{x} F(\vec{u}(\tau)) d \tau\right\|_{\mathbb{H}^{s}} \leq \frac{K_{1}}{2 \mu} \int_{0}^{2 \mu \tau} \sqrt{1+\frac{1}{r}} d r \sup _{\tau \in[0, T]}\left\|\partial_{x} F(\vec{u}(\tau))\right\|_{\mathbb{H}^{s-1}}<\infty .
$$

Hence, $\Theta \vec{u}(t) \in \mathbb{H}^{s}$ for all $t \in[0, T]$.

Now let's consider the continuity. For $\left.\left.t_{0} \in\right] 0, T\right]$ suppose that $t<t_{0}$, in this way

$$
\begin{aligned}
\left\|\Theta \vec{u}(t)-\Theta \vec{u}\left(t_{0}\right)\right\|_{\mathbb{H}^{s}} \leq & \left\|\vec{W}_{\mu}(t) \vec{\phi}-\vec{W}_{\mu}\left(t_{0}\right) \vec{\phi}\right\|_{\mathbb{H}^{s}} \\
& +\int_{0}^{t}\left\|\left[\vec{W}_{\mu}(t-\tau)-\vec{W}_{\mu}\left(t_{0}-\tau\right)\right] \partial_{x} F(\vec{u}(\tau))\right\|_{\mathbb{H}^{s}} d \tau \\
& +\int_{t}^{t_{0}}\left\|\vec{W}_{\mu}\left(t_{0}-\tau\right) \partial_{x} F(\vec{u}(\tau))\right\|_{\mathbb{H}^{s}} d \tau .
\end{aligned}
$$

Then, due to the strong continuity of the semigroup $\left\{\vec{W}_{\mu}(t)\right\}_{t \geq 0}$ we have

$$
\left\|\vec{W}_{\mu}\left(t_{0}-\tau\right) \partial_{x} F(\vec{u}(\tau))\right\|_{\mathbb{H}^{s}} \leq \sup _{\left[0, t_{0}\right]}\left\|\vec{W}_{\mu}\left(t_{0}-\tau\right) \partial_{x} F(\vec{u}(\tau))\right\|_{\mathbb{H}^{s}},
$$

then

$$
\begin{aligned}
\left\|\Theta \vec{u}(t)-\Theta \vec{u}\left(t_{0}\right)\right\|_{\mathbb{H}^{s}} \leq & \left\|\vec{W}_{\mu}(t) \vec{\phi}-\vec{W}_{\mu}\left(t_{0}\right) \vec{\phi}\right\|_{\mathbb{H}^{s}} \\
& +\int_{0}^{t}\left\|\left[\vec{W}_{\mu}(t-\tau)-\vec{W}_{\mu}\left(t_{0}-\tau\right)\right] \partial_{x} F(\vec{u}(\tau))\right\|_{\mathbb{H}^{s}} d \tau \\
& +\left|t-t_{0}\right| \sup _{\left[0, t_{0}\right]}\left\|\vec{W}_{\mu}\left(t_{0}-\tau\right) \partial_{x} F(\vec{u}(\tau))\right\|_{\mathbb{H}^{s}} .
\end{aligned}
$$

The first summation of the second member of (3.5) converges to zero when $t \rightarrow t_{0}^{-}$by the continuity of the application $\vec{W}_{\mu}(\cdot) \Phi: \mathbb{R}_{0}^{+} \rightarrow \mathbb{H}^{s}$ shown in the theorem 2.2 . The same happens with the second summing for the same reason and Lebesgue's dominated convergence theorem. The last addition converges trivially to zero when $t \rightarrow t_{0}^{-}$. This shows us the continuity to the left of $t_{0}$. The continuity to the right of $t_{0}$ is followed in an analogous way, and hence the continuity in $t_{0}$.

Let's see now, there is $T_{1}=T_{1}\left(\|\vec{\phi}\|_{\mathbb{H}^{s}}, \alpha, \beta, s, \mu\right) \in[0, T]$ such that $\Theta$ defined on $\mathcal{E}_{s}\left(T_{1}\right)$ and it has range $\mathcal{R}(\Theta)$ contained in $\mathcal{E}_{s}\left(T_{1}\right)$. Let be $T>0$ and $\vec{u} \in \mathcal{E}_{s}(T)$, then for $0 \leq t \leq T$, we have 


$$
\left\|\Theta \vec{u}(t)-\vec{W}_{\mu}(t) \vec{\phi}\right\|_{\mathbb{H}^{s}} \leq \int_{0}^{t}\left\|\vec{W}_{\mu}(t-\tau) \partial_{x} F(\vec{u}(\tau))\right\|_{\mathbb{H}^{s}} d \tau
$$

Using the equality (2.7) with $\sigma=1$ we have

$$
\left\|\vec{W}_{\mu}(t-\tau) \partial_{x} F(\vec{u}(\tau))\right\|_{\mathbb{H}^{s}} \leq K_{1} \sqrt{1+\frac{1}{2 \mu(t-\tau)}}\left\|\partial_{x} F(\vec{u}(\tau))\right\|_{\mathbb{H}^{s-1}},
$$

then

$$
\left\|\Theta \vec{u}(t)-\vec{W}_{\mu}(t) \vec{\phi}\right\|_{\mathbb{H}^{s}} \leq K_{1} \int_{0}^{t} \sqrt{1+\frac{1}{2 \mu(t-\tau)}}\left\|\partial_{x} F(\vec{u}(\tau))\right\|_{\mathbb{H}^{s-1}} d \tau .
$$

But, because of the triangular inequality and because $H^{s}$ is a Banach algebra, we have

$$
\begin{aligned}
\left\|\partial_{x} F(\vec{u}(\tau))\right\|_{\mathbb{H}^{s-1}} \leq & \left(\frac{|\alpha(t)|}{p+1}\left\|u^{p+1}\right\|_{H^{s}}+\frac{1}{p+1}\left\|v^{p+1}\right\|_{H^{s}}+\left\|u v^{p}\right\|_{H^{s}}\right) \\
& +\left(\frac{1}{p+1}\left\|u^{p+1}\right\|_{H^{s}}+\frac{|\beta(t)|}{p+1}\left\|v^{p+1}\right\|_{H^{s}}+\left\|u^{p} v\right\|_{H^{s}}\right) \\
\leq & \frac{1+\|\alpha(t)\|_{L^{\infty}[0, T]}}{p+1}\|u\|_{H^{s}}^{p+1}+\frac{1+\|\beta(t)\|_{L^{\infty}[0, T]}}{p+1}\|v\|_{H^{s}}^{p+1}+\|u\|_{H^{s}}\|v\|_{H^{s}}^{p}+\|u\|_{H^{s}}^{p}\|v\|_{H^{s}} \\
\leq & C\left(\|u\|_{H^{s}}^{p+1}+\|v\|_{H^{s}}^{p+1}+\|u\|_{H^{s}}\|v\|_{H^{s}}^{p}+\|u\|_{H^{s}}^{p}\|v\|_{H^{s}}\right) \\
\leq & C\|\vec{u}\|_{\mathbb{H}^{s}}^{p+1},
\end{aligned}
$$

where $C=C(\alpha, \beta, s)$. We note that if $\vec{u} \in \mathcal{E}_{s}(T)$ and as $\left\{\vec{W}_{\mu}(t)\right\}_{t \geq 0}$ is a semigroup of contractions by theorem 2.2, we have

$$
\|\vec{u}\|_{\mathbb{H}^{s}} \leq\left\|\vec{u}-\vec{W}_{\mu}(t) \vec{\phi}\right\|_{\mathbb{H}^{s}}+\left\|\vec{W}_{\mu}(t) \vec{\phi}\right\|_{\mathbb{H}^{s}} \leq\|\vec{\phi}\|_{\mathbb{H}^{s}}+\|\vec{\phi}\|_{\mathbb{H}^{s}}=2\|\vec{\phi}\|_{\mathbb{H}^{s}}
$$

and using this inequality in (3.7) we obtain

$$
\left\|\partial_{x} F(\vec{u}(\tau))\right\|_{\mathbb{H}^{s-1}} \leq C\|\vec{\phi}\|_{\mathbb{H}^{s}}^{p+1}
$$

Then, from (3.6) and changing the variable we get

$$
\left\|\Theta \vec{u}(t)-\vec{W}_{\mu}(t) \vec{\phi}\right\|_{\mathbb{H}^{s}} \leq C\|\vec{\phi}\|_{\mathbb{H}^{s}}^{p+1} \int_{0}^{t} \sqrt{1+\frac{1}{2 \mu(t-\tau)}} d \tau \leq C\|\vec{\phi}\|_{\mathbb{H}^{s}}^{p+1} \int_{0}^{2 \mu t} \sqrt{1+\frac{1}{\tau}} d \tau,
$$

where $C=C(\alpha, \beta, s, \mu)$. We note that

$$
\int_{0}^{2 \mu t} \sqrt{1+\frac{1}{\tau}} d \tau \leq 2(\mu t+\sqrt{2 \mu t})
$$

then

$$
\lim _{t \rightarrow 0^{+}} C\|\vec{\phi}\|_{\mathbb{H}^{s}}^{p} \int_{0}^{2 \mu t} \sqrt{1+\frac{1}{\tau}} d \tau=0
$$

imply that there exists $T_{1}=T_{1}\left(\|\vec{\phi}\|_{\mathbb{H}^{s}}, \alpha, \beta, s, \mu\right)$ such that $C\|\vec{\phi}\|_{\mathbb{H}^{s}}^{p} \int_{0}^{2 \mu T_{1}} \sqrt{1+\frac{1}{\tau}} d \tau \leq 1$ if $0<t<T_{1}$.

Thus, in inequality (3.9) for $0<t<T_{1}$ we have

$$
\left\|\Theta \vec{u}(t)-\vec{W}_{\mu}(t) \vec{\phi}\right\|_{\mathbb{H}^{s}} \leq\|\vec{\phi}\|_{\mathbb{H}^{s}} .
$$

That is to say, $\Theta \vec{u}(t) \in \mathcal{E}_{s}\left(T_{1}\right)$. Therefore, there exists $T_{1}=T_{1}\left(\|\vec{\phi}\|_{\mathbb{H}^{s}}, \alpha, \beta, s, \mu\right) \in[0, T]$ such that $\mathcal{R}(\Theta) \subseteq$ $\mathcal{E}_{s}\left(T_{1}\right)$. 
We will now prove that there exists $T_{\mu}=T_{\mu}\left(\|\vec{\phi}\|_{\mathbb{H}^{s}}, \alpha, \beta, s, \mu\right) \in\left[0, T_{1}\right]$ such that $\Theta: \mathcal{E}_{s}\left(T_{\mu}\right) \rightarrow \mathcal{E}_{s}\left(T_{\mu}\right)$ is a contraction. Of course, if $\vec{u}=\left(u_{1}, v_{1}\right), \vec{v}=\left(u_{2}, v_{2}\right) \in \mathcal{E}_{s}\left(T_{1}\right)$ then

$$
\|\Theta \vec{u}(t)-\Theta \vec{v}(t)\|_{\mathbb{H}^{s}} \leq \int_{0}^{t}\left\|\vec{W}_{\mu}(t-\tau)\left[\partial_{x} F(\vec{u}(\tau))-\partial_{x} F(\vec{v}(\tau))\right]\right\|_{\mathbb{H}^{s}} d \tau .
$$

For the equality (2.7) with $\sigma=1$,

$$
\|\Theta \vec{u}(t)-\Theta \vec{v}(t)\|_{\mathbb{H}^{s}} \leq K_{1} \int_{0}^{t} \sqrt{1+\frac{1}{2 \mu(t-\tau)}}\left\|\partial_{x} F(\vec{u}(\tau))-\partial_{x} F(\vec{v}(\tau))\right\|_{\mathbb{H}^{s-1}} d \tau .
$$

On the other hand, using the factorizing, the triangular inequality and the algebra properties of $H^{s}$, we obtain

$$
\begin{aligned}
\left\|\partial_{x} F(\vec{u}(\tau))-\partial_{x} F(\vec{v}(\tau))\right\|_{\mathbb{H}^{s-1}} & \frac{1+\|\alpha(t)\|_{L^{\infty}[0, T]}}{p+1}\left\|u_{1}^{p+1}-u_{2}^{p+1}\right\|_{H^{s}}+\frac{1+\|\beta(t)\|_{L^{\infty}[0, T]}}{p+1}\left\|v_{1}^{p+1}-v_{2}^{p+1}\right\|_{H^{s}} \\
& \quad+\left\|u_{1} v_{1}^{p}-u_{2} v_{2}^{p}\right\|_{H^{s}}+\left\|u_{1}^{p} v_{1}-u_{2}^{p} v_{2}\right\|_{H^{s}} \\
\leq & C\left\|u_{1}-u_{2}\right\|_{H^{s}}\left(\sum_{j=1}^{p+1}\left\|u_{1}\right\|_{H^{s}}^{p+1-j}\left\|u_{2}\right\|_{H^{s}}^{j-1}+\left\|v_{2}\right\|_{H^{s}} \sum_{j=1}^{p}\left\|u_{1}\right\|_{H^{s}}^{p-j}\left\|u_{2}\right\|_{H^{s}}^{j-1}+\left\|v_{2}\right\|_{H^{s}}^{p}\right) \\
& \quad+C\left\|v_{1}-v_{2}\right\|_{H^{s}}\left(\sum_{j=1}^{p+1}\left\|v_{1}\right\|_{H^{s}}^{p+1-j}\left\|v_{2}\right\|_{H^{s}}^{j-1}+\left\|u_{1}\right\|_{H^{s}} \sum_{j=1}^{p}\left\|v_{1}\right\|_{H^{s}}^{p-j}\left\|v_{2}\right\|_{H^{s}}^{j-1}+\left\|u_{1}\right\|_{H^{s}}^{p}\right)
\end{aligned}
$$

where $C=C(\alpha, \beta, s)$. Inequalities $\max \left\{\left\|u_{1}\right\|_{H^{s}},\left\|v_{1}\right\|_{H^{s}}\right\} \leq\|\vec{u}\|_{\mathbb{H}^{s}}, \max \left\{\left\|u_{2}\right\|_{H^{s}},\left\|v_{2}\right\|_{H^{s}}\right\} \leq\|\vec{v}\|_{\mathbb{H}^{s}}$ and (3.8) implies that

$$
\left\|\partial_{x} F(\vec{u}(\tau))-\partial_{x} F(\vec{v}(\tau))\right\|_{\mathbb{H}^{s-1}} \leq C\|\vec{\phi}\|_{\mathbb{H}^{s}}^{p}\left(\left\|u_{1}-u_{2}\right\|_{H^{s}}+\left\|v_{1}-v_{2}\right\|_{H^{s}}\right) .
$$

Since $\left\|u_{1}-u_{2}\right\|_{H^{s}}+\left\|v_{1}-v_{2}\right\|_{H^{s}} \leq\|\vec{u}-\vec{v}\|_{\mathbb{H}^{s}}$ we obtain

$$
\left\|\partial_{x} F(\vec{u}(\tau))-\partial_{x} F(\vec{v}(\tau))\right\|_{\mathbb{H}^{s-1}} \leq C\|\vec{\phi}\|_{\mathbb{H}^{s}}^{p}\|\vec{u}(\tau)-\vec{v}(\tau)\|_{\mathbb{H}^{s}}
$$

Then of (3.11) we have

$$
\begin{aligned}
\|\Theta \vec{u}(t)-\Theta \vec{v}(t)\|_{\mathbb{H}^{s}} & \leq C\|\vec{\phi}\|_{\mathbb{H}^{s}} \int_{0}^{t} \sqrt{1+\frac{1}{2 \mu(t-\tau)}}\|\vec{u}(\tau)-\vec{v}(\tau)\|_{\mathbb{H}^{s}} d \tau \\
& \leq C\|\vec{\phi}\|_{\mathbb{H}^{s}} \int_{0}^{2 \mu t} \sqrt{1+\frac{1}{\tau}} d \tau d(\vec{u}, \vec{v}) .
\end{aligned}
$$

where $C=C(\alpha, \beta, s, \mu)$. Taking the supremum in $\left[0, T_{1}\right]$ we obtain

$$
d(\Theta \vec{u}, \Theta \vec{v})=\sup _{0 \leq t \leq T_{1}}\|(\Theta \vec{u})(t)-(\Theta \vec{v})(t)\|_{\mathbb{H}^{s}} \leq C\|\vec{\phi}\|_{\mathbb{H}^{s}} \int_{0}^{2 \mu T_{1}} \sqrt{1+\frac{1}{\tau}} d \tau d(\vec{u}, \vec{v}) .
$$

Now, as $\lim _{T_{1} \rightarrow 0^{+}} C\|\vec{\phi}\|_{\mathbb{H}^{s}} \int_{0}^{2 \mu T_{1}} \sqrt{1+\frac{1}{\tau}} d \tau=0$, it follows the existence of $\left.\left.T_{\mu}=T_{\mu}\left(\|\vec{\phi}\|_{\mathbb{H}^{s}}, \alpha, \beta, s, \mu\right) \in\right] 0, T_{1}\right]$ such that

$$
0<\lambda=C\|\vec{\phi}\|_{\mathbb{H} s} \int_{0}^{2 \mu T_{\mu}} \sqrt{1+\frac{1}{\tau}} d \tau<1
$$

Thus, we conclude that

$$
d(\Theta \vec{u}, \Theta \vec{v}) \leq \lambda d(\vec{u}, \vec{v}) \text { with } 0<\lambda<1,
$$

and we conclude that $\Theta$ is a contraction.

Theorem 3.2. If $\alpha, \beta \in C(\mathbb{R}) \cap L^{\infty}(\mathbb{R}), \mu>0$ and $\vec{\phi} \in \mathbb{H}^{s}, s>\frac{3}{2}$, there exists $T_{\mu}=T_{\mu}\left(\|\vec{\phi}\|_{\mathbb{H}^{s}}, \alpha, \beta, s, \mu\right)>$ 0 and the function

$$
\vec{u}_{\mu} \in C\left(\left[0, T_{\mu}\right]: \mathbb{H}^{s}\right)
$$


as a unique real solution of the integral equation (3.3).

Proof. By the proposition 3.1 the hypotheses of the Banach fixed-point theorem are satisfied, then there exists a unique $\vec{u}_{\mu} \in \mathcal{E}_{s}\left(T_{\mu}\right) \subset C\left(\left[0, T_{\mu}\right]: \mathbb{H}^{s}\right)$ such that $\Theta \vec{u}_{\mu}=\vec{u}_{\mu}$, that is,

$$
\Theta \vec{u}_{\mu}(t)=\vec{W}_{\mu}(t) \vec{\phi}-\int_{0}^{t} \vec{W}_{\mu}(t-\tau) \partial_{x} F(\vec{u}(\tau)) d \tau=\vec{u}_{\mu}(t)
$$

for all $t \in\left[0, T_{\mu}\right]$.

For uniqueness, let $\vec{u}$ and $\vec{v}$ be two solutions in $C\left(\left[0, T_{\mu}\right]: \mathbb{H}^{s}\right)$ of the integral equation (3.3). For any $t \in\left[0, T_{\mu}\right]$, by the inequality (2.7) with $\sigma=1,(3.12)$, a change of variable and (3.10), we have

$$
\begin{aligned}
\|\vec{u}(t)-\vec{v}(t)\|_{\mathbb{H}^{s}} & \leq \int_{0}^{t}\left\|\vec{W}_{\mu}(t-\tau)\left[\partial_{x} F(\vec{u}(\tau))-\partial_{x} F(\vec{v}(\tau))\right]\right\|_{\mathbb{H}^{s}} d \tau \\
& \leq \int_{0}^{t} K_{1} \sqrt{1+\frac{1}{2 \mu(t-\tau)}}\left\|\partial_{x} F(\vec{u}(\tau))-\partial_{x} F(\vec{v}(\tau))\right\|_{\mathbb{H}^{s-1}} d \tau \\
& \leq \frac{C}{2 \mu}\|\vec{\phi}\|_{\mathbb{H}^{s}}^{p} \sup _{\tau \in[0, t]}\|\vec{u}(\tau)-\vec{v}(\tau)\|_{\mathbb{H}^{s}} \int_{0}^{2 \mu t} \sqrt{1+\frac{1}{\tau}} d \tau \\
& \leq \kappa(t) \sup _{\tau \in[0, t]}\|\vec{u}(\tau)-\vec{v}(\tau)\|_{\mathbb{H}^{s}},
\end{aligned}
$$

where $\kappa(t)=\frac{C}{\mu}\|\vec{\phi}\|_{\mathbb{H}^{s}}^{p}(\mu t+\sqrt{2 \mu t})$. Since $\kappa$ is continue and increasing in $\left[0,+\infty\left[, \kappa(0)=0\right.\right.$ and $\lim _{t \rightarrow+\infty} \kappa(t)=$ $+\infty$, by the mean value theorem, there exists $T^{*}>0$ such that $\kappa\left(T^{*}\right)=\frac{1}{2}$. Let be $T_{1}=\min \left\{T_{\mu}, T^{*}\right\}$, then $\kappa(t) \leq \kappa\left(T_{1}\right) \leq k\left(T^{*}\right)=\frac{1}{2}$ and for $t \in\left[0, T_{1}\right]$ we have

$$
\|\vec{u}(t)-\vec{v}(t)\|_{\mathbb{H}^{s}} \leq \frac{1}{2} \sup _{\tau \in[0, t]}\|\vec{u}(\tau)-\vec{v}(\tau)\|_{\mathbb{H}^{s}} \leq \frac{1}{2} \sup _{\tau \in\left[0, T_{1}\right]}\|\vec{u}(\tau)-\vec{v}(\tau)\|_{\mathbb{H}^{s}},
$$

and taking the supremun on $\left[0, T_{1}\right]$, we obtain

$$
\sup _{t \in\left[0, T_{1}\right]}\|\vec{u}(t)-\vec{v}(t)\|_{\mathbb{H}^{s}} \leq \frac{1}{2} \sup _{\tau \in\left[0, T_{1}\right]}\|\vec{u}(\tau)-\vec{v}(\tau)\|_{\mathbb{H}^{s}} .
$$

Therefore, $\sup _{t \in\left[0, T_{1}\right]}\|\vec{u}(t)-\vec{v}(t)\|_{\mathbb{H}^{s}} \leq 0$ and thus $\vec{u}=\vec{v}$ in $\left[0, T_{1}\right]$.

If $T_{1}=T_{\mu}$ we get the uniqueness, but if $T_{1}=T^{*}$ and defining $T_{2}=\min \left\{T_{\mu}, 2 T^{*}\right\}$ it follows that $T_{1}<T_{2}$. Then, for $t \in\left[T_{1}, T_{2}\right]$ we obtain

$$
\begin{aligned}
\|\vec{u}(t)-\vec{v}(t)\|_{\mathbb{H}^{s}} & \leq C\|\vec{\phi}\|_{\mathbb{H}^{s}}^{p} \int_{T_{1}}^{t} \sqrt{1+\frac{1}{2 \mu(t-\tau)}}\|\vec{u}(\tau)-\vec{v}(\tau)\|_{\mathbb{H}^{s}} d \tau \\
& \leq \frac{C}{\mu}\|\vec{\phi}\|_{\mathbb{H}^{s}}^{p} \sup _{\tau \in[0, t]}\|\vec{u}(\tau)-\vec{v}(\tau)\|_{\mathbb{H}^{s}} \int_{0}^{2 \mu\left(t-T_{1}\right)} \sqrt{1+\frac{1}{\tau}} d \tau \\
& \leq \kappa\left(t-T_{1}\right) \sup _{\tau \in\left[0, T_{2}\right]}\|\vec{u}(\tau)-\vec{v}(\tau)\|_{\mathbb{H}^{s}} \\
& \leq \kappa\left(T_{2}-T_{1}\right) \sup _{\tau \in\left[0, T_{2}\right]}\|\vec{u}(\tau)-\vec{v}(\tau)\|_{\mathbb{H}^{s}}
\end{aligned}
$$

because of $T_{2} \leq 2 T^{*}, T_{1}=T^{*}$, we have

$$
\kappa\left(T_{2}-T_{1}\right) \leq \kappa\left(T^{*}\right)
$$

Thus from (3.14) we get

$$
\|\vec{u}(t)-\vec{v}(t)\|_{\mathbb{H}^{s}} \leq \kappa\left(T^{*}\right) \sup _{\tau \in\left[0, T_{2}\right]}\|\vec{u}(\tau)-\vec{v}(\tau)\|_{\mathbb{H}^{s}}=\frac{1}{2} \sup _{\tau \in\left[0, T_{2}\right]}\|\vec{u}(\tau)-\vec{v}(\tau)\|_{\mathbb{H}^{s}} .
$$

Taking the supremum on $\left[0, T_{2}\right]$ we obtain (3.13) in $\left[0, T_{2}\right]$, then $\vec{u}=\vec{v}$ in $\left[0, T_{2}\right]$. If $T_{2}=T_{\mu}$ we obtain the uniqueness, otherwise we repeat the process for $T_{3}=\min \left\{T_{\mu}, 3 T^{*}\right\}$. Since $\left[0, T_{\mu}\right]$ is compact, there exists $n \in \mathbb{N}$ such that $T_{\mu} \leq n T^{*}$, hence $\vec{u}=\vec{v}$ in $\left[0, T_{\mu}\right]$. 
As a consequence of the regularizing property of the semigroup $\left\{\vec{W}_{\mu}(t)\right\}_{t \geq 0}$ proved in the theorem 2.2, we will show that the solution of the integral equation (3.3), obtained in the theorem 3.2, is more regular than the initial data, that is, if $\vec{\phi} \in \mathbb{H}^{s}$ with $s>\frac{3}{2}$, then $\left.\left.\vec{u}_{\mu}:\right] 0, T_{\mu}\right] \rightarrow \mathbb{H}^{s+r}$ is continue for all $r \geq 0$.

Theorem 3.3. Let $\alpha, \beta \in C(\mathbb{R}) \cap L^{\infty}(\mathbb{R}), \mu>0$ and $\vec{\phi} \in \mathbb{H}^{s}, s>\frac{3}{2}$ be given. The function $\vec{u}_{\mu}$ of the theorem 3.2 satisfies

$$
\left.\left.\vec{u}_{\mu} \in C(] 0, T_{\mu}\right]: \mathbb{H}^{s+r}\right)
$$

for all $r \geq 0$.

Proof. Let $\left.t \in] 0, T_{\mu}\right]$ and we consider the integral equation

$$
\vec{u}_{\mu}(t)=\vec{W}_{\mu}(t) \vec{\phi}-\int_{0}^{t} \vec{W}_{\mu}(t-\tau) \partial_{x} F\left(\vec{u}_{\mu}(\tau)\right) d \tau \equiv \vec{W}_{\mu}(t) \vec{\phi}-G(t) .
$$

From the theorem 2.2 we deduce the continuity of the application $t \in\left[0,+\infty\left[\mapsto \vec{W}_{\mu}(t) \vec{\phi} \in \mathbb{H}^{s+r}\right.\right.$ for all $r \geq 0$, that is, $\left.\left.\vec{W}_{\mu}(\cdot) \vec{\phi} \in C(] 0, T_{\mu}\right]: \mathbb{H}^{s+r}\right)$.

Suppose that $0 \leq r<1$, then

$$
\|G(t)\|_{\mathbb{H}^{s+r}} \leq \int_{0}^{t}\left\|\vec{W}_{\mu}(t-\tau) \partial_{x} F\left(\vec{u}_{\mu}(\tau)\right)\right\|_{\mathbb{H}^{s+r}} d \tau .
$$

Using (2.7) with $\sigma=r+1$ we have,

$$
\left\|\vec{W}_{\mu}(t-\tau) \partial_{x} F\left(\vec{u}_{\mu}(\tau)\right)\right\|_{\mathbb{H}^{s+r}} \leq K_{r+1} \sqrt{1+\left(\frac{1}{2 \mu(t-\tau)}\right)^{r+1}}\left\|\partial_{x} F\left(\vec{u}_{\mu}(\tau)\right)\right\|_{\mathbb{H}^{s-1}},
$$

and from (3.7) we obtain

$$
\left\|\vec{W}_{\mu}(t-\tau) \partial_{x} F\left(\vec{u}_{\mu}(\tau)\right)\right\|_{\mathbb{H}^{s+r}} \leq C \sqrt{1+\left(\frac{1}{2 \mu(t-\tau)}\right)^{r+1}} \sup _{0 \leq t \leq \tau}\left\|\vec{u}_{\mu}(\tau)\right\|_{\mathbb{H}^{s}}^{p+1}
$$

Thus we get in (3.16),

$$
\|G(t)\|_{\mathbb{H}^{s+r}} \leq C \sup _{0 \leq \tau \leq T_{\mu}}\left\|\vec{u}_{\mu}(\tau)\right\|_{\mathbb{H}^{s}}^{p+1} \int_{0}^{2 \mu t} \sqrt{1+\frac{1}{\tau^{r+1}}} d \tau
$$

Then, by $\frac{r+1}{2} \in\left[0,1\left[\right.\right.$, the integral $\int_{0}^{2 \mu t} \sqrt{1+\frac{1}{\tau^{r+1}}} d \tau$ is convergent, in consequence $G(t) \in \mathbb{H}^{s+r}$ for any $r \in[0,1[$.

Now we show that $\left.\left.G \in C(] 0, T_{\mu}\right]: \mathbb{H}^{s+r}\right)$ whenever $r \in\left[0,1[\right.$. To this end, we consider $\left.t \in] 0, T_{\mu}\right]$ and $h>0$ such that $t+h$ in $\left.] 0, T_{\mu}\right]$, then

$$
\begin{aligned}
\|G(t+h)-G(t)\|_{\mathbb{H}^{s+r}} \leq & \int_{t}^{t+h}\left\|\vec{W}_{\mu}(t+h-\tau) \partial_{x} F\left(\vec{u}_{\mu}(\tau)\right)\right\|_{\mathbb{H}^{s+r}} d \tau \\
& +\int_{0}^{t}\left\|\left[\vec{W}_{\mu}(t+h-\tau)-\vec{W}_{\mu}(t-\tau)\right] \partial_{x} F\left(\vec{u}_{\mu}(\tau)\right)\right\|_{\mathbb{H}^{s+r}} d \tau
\end{aligned}
$$

Using (2.7) with $\sigma=r+1$, by (3.7) and changing the variable, we have

$$
\begin{aligned}
\int_{t}^{t+h} \| \vec{W}_{\mu}(t+h-\tau) \partial_{x} F\left(\vec{u}_{\mu}(\tau)\right) & \|_{\mathbb{H}^{s+r}} d \tau \\
& \leq K_{r+1} \int_{t}^{t+h} \sqrt{1+\left(\frac{1}{2 \mu(t+h-\tau)}\right)^{r+1}}\left\|\vec{u}_{\mu}(\tau)\right\|_{\mathbb{H}^{s}}^{2} d t \\
& \leq C \sup _{\left.\tau \in] 0, T_{\mu}\right]}\|\vec{u}(\tau)\|_{\mathbb{H}^{s}}^{2} \int_{t}^{t+h} \sqrt{1+\left(\frac{1}{2 \mu(t+h-\tau)}\right)^{r+1}} d t \\
& \leq C \sup _{\left.\tau \in] 0, T_{\mu}\right]}\left\|\vec{u}_{\mu}(\tau)\right\|_{\mathbb{H}^{s}}^{2} \int_{0}^{2 \mu h} \sqrt{1+\frac{1}{\tau^{r+1}}} d \tau .
\end{aligned}
$$


As $\lim _{h \rightarrow 0^{+}} \int_{0}^{2 \mu h} \sqrt{1+\frac{1}{\tau^{r+1}}} d \tau=0$ because of $\frac{r+1}{2} \in[0,1[$, we get

$$
\lim _{h \rightarrow 0^{+}} \int_{t}^{t+h}\left\|\vec{W}_{\mu}(t+h-\tau) F\left(\vec{u}_{\mu}(\tau)\right)\right\|_{\mathbb{H}^{s+r}} d \tau=0 .
$$

On the other hand, the continuity of the application $t \in\left[0,+\infty\left[\mapsto \vec{W}_{\mu}(t) \vec{\phi} \in \mathbb{H}^{s+r}\right.\right.$ for all $r \geq 0$, the inequality (2.7) and $\left.\left.\sqrt{1+\frac{1}{(\cdot)^{r+1}}} \in L^{1}(] 0,2 \mu t\right]: \mathbb{R}\right)$, by the dominated convergence theorem, implies that

$$
\lim _{h \rightarrow 0^{+}} \int_{0}^{t}\left\|\left[\vec{W}_{\mu}(t+h-\tau)-\vec{W}_{\mu}(t-\tau)\right] \partial_{x} F\left(\vec{u}_{\mu}(\tau)\right)\right\|_{\mathbb{H}^{s+r}} d \tau=0
$$

Thus in (3.17) we have

$$
\lim _{h \rightarrow 0^{+}}\|G(t+h)-G(t)\|_{\mathbb{H}^{s+r}}=0,
$$

so we show that $G$ is continuous by the right of $t$. In the same way the continuity by the left of $t$ is proved. Hence, from (3.15), we proved that $\left.\left.\vec{u}_{\mu} \in C(] 0, T_{\mu}\right]: \mathbb{H}^{s+r}\right)$ if $0 \leq r<1$.

Using the results proven and the same procedure, we can show that $\left.\left.G \in C(] 0, T_{\mu}\right]: \mathbb{H}^{s+2 r}\right)$ and from there $\left.\left.\vec{u}_{\mu} \in C(] 0, T_{\mu}\right]: \mathbb{H}^{s+2 r}\right)$. An inductive argument proves that $\left.\left.G \in C(] 0, T_{\mu}\right]: \mathbb{H}^{s+n r}\right)$ for each $n \in \mathbb{N}$ and thence follows the thesis of the theorem.

This result will be fundamental to show that the existence interval of the solution of the regularized system is independent of $\mu$, as we will see in the theorem 4.1.

4. Existence and uniqueness of local solution. In this section we will prove that the initial value problem regularized (3.1) is well possed locally. For this we will first prove that the function $\vec{u}_{\mu}$ of the theorem 3.2 is the unique solution of the regularized problem in $\mathbb{H}^{s-2}$. The proof is based on the work of R. Iório [9] and the theorem 2.2.

Theorem 4.1. Let $\alpha, \beta \in C(\mathbb{R}) \cap L^{\infty}(\mathbb{R}), \mu>0$ and $\Phi \in \mathbb{H}^{s}, s>\frac{3}{2}$ be given, then the function $\vec{u}_{\mu}$ of the theorem 3.2 satisfies

$$
\vec{u}_{\mu} \in C\left(\left[0, T_{\mu}\right]: \mathbb{H}^{s}\right) \cap C^{1}\left(\left[0, T_{\mu}\right]: \mathbb{H}^{s-2}\right)
$$

and is the unique solution of (3.1). Moreover, for all $r \geq 0$

$$
\left.\left.\vec{u}_{\mu} \in C(] 0, T_{\mu}\right]: \mathbb{H}^{s+r}\right) \cap C^{1}\left(\left[0, T_{\mu}\right]: \mathbb{H}^{s+r-2}\right) .
$$

Proof. Let's see the existence of a solution. From the theorem 2.2 and the theory of semigroups we have,

$$
\partial_{t} \vec{W}_{\mu}(t) \vec{\phi}=-A_{\mu} \vec{W}_{\mu}(t) \vec{\phi},
$$

for $t>0$ in $\mathbb{H}^{\mathbf{s}-\mathbf{2}}$. For $\mu>0$, we consider

$$
G(t)=\int_{0}^{t} \vec{W}_{\mu}(t-\tau) \partial_{x} F\left(\vec{u}_{\mu}(\tau)\right) d \tau
$$

For $0 \leq t<T_{\mu}$ and $h>0$ such that $\left.\left.t+h \in\right] 0, T_{\mu}\right]$ it follows that,

$$
\frac{G(t+h)-G(t)}{h}=\frac{\vec{W}_{\mu}(h)-I}{h} \int_{0}^{t} \vec{W}_{\mu}(t-\tau) \partial_{x} F\left(\vec{u}_{\mu}(\tau)\right) d \tau+\vec{W}_{\mu}\left(t+h-c_{h}\right) \partial_{x} F\left(\vec{u}_{\mu}\left(c_{h}\right)\right),
$$

where it has been used that $\vec{W}_{\mu}(h)-I$ is a linear operator and the mean value theorem for Bochner integrals in the interval $[t, t+h]$ with $c_{h} \in[t, t+h]$.

As $-A_{\mu}$ is the generator of the semigroup $\left\{\vec{W}_{\mu}(t)\right\}_{t \geq 0}$ we have

$$
\lim _{h \rightarrow 0^{+}} \frac{\vec{W}_{\mu}(h)-I}{h} \int_{0}^{t} \vec{W}_{\mu}(t-\tau) \partial_{x} F\left(\vec{u}_{\mu}(\tau)\right) d \tau=-A_{\mu} \int_{0}^{t} \vec{W}_{\mu}(t-\tau) \partial_{x} F\left(\vec{u}_{\mu}(\tau)\right) d \tau .
$$

In addition, it follows from $c_{h} \in[t, t+h]$ that if $h \rightarrow 0^{+}$then $c_{h} \rightarrow t$, hence

$$
\lim _{h \rightarrow 0^{+}} \vec{W}_{\mu}\left(t+h-c_{h}\right) F\left(\vec{u}_{\mu}\left(c_{h}\right)\right)=\vec{W}_{\mu}(0) F\left(\vec{u}_{\mu}(t)\right)=F\left(\vec{u}_{\mu}(t)\right) .
$$


Thus, we get

$$
\partial_{t}^{+} G(t)=-A_{\mu} \int_{0}^{t} \vec{W}_{\mu}(t-\tau)\left(F \vec{u}_{\mu}(\tau) d \tau+F\left(\vec{u}_{\mu}(t)\right)=-A_{\mu} G(t)+F\left(\vec{u}_{\mu}(t)\right)\right.
$$

Similarly, for $h<0$ we obtain

$$
\partial_{t}^{-} G(t)=-A_{\mu} \int_{0}^{t} \vec{W}_{\mu}(t-\tau) F\left(\vec{u}_{\mu}(\tau)\right) d \tau+F\left(\vec{u}_{\mu}(t)\right)=-A_{\mu} G(t)+F\left(\vec{u}_{\mu}(t)\right)
$$

For this, it is enough to take $s=-h>0$ and consider $\partial_{t}^{-} G(t)=\lim _{s \rightarrow 0^{+}} \frac{G(t)-G(t-s)}{s}$.

Thus $\partial_{t} G(t)=\partial_{t}^{+} G(t)=\partial_{t}^{-} G(t)$ and

$$
\partial_{t} \vec{u}_{\mu}(t)=\partial_{t}\left(\vec{W}_{\mu}(t) \Phi-G(t)\right)=-A_{\mu} \vec{u}_{\mu}(t)-F\left(\vec{u}_{\mu}(t)\right) .
$$

Then, since $\vec{u}_{\mu} \in C\left(\left[0, T_{\mu}\right]: \mathbb{H}^{s}\right)$ satisfies (3.1), and given that $-A_{\mu} \in \mathcal{L}\left(\mathbb{H}^{s}, \mathbb{H}^{s-2}\right)$ and $-F\left(\vec{u}_{\mu}\right) \in C\left(\left[0, T_{\mu}\right]: \mathbb{H}^{s-1}\right) \subseteq$ $C\left(\left[0, T_{\mu}\right]: \mathbb{H}^{s-2}\right)$, it follows that $\vec{u}_{\mu} \in C^{1}\left(\left[0, T_{\mu}\right], \mathbb{H}^{s-2}\right)$.

For uniqueness, let

$$
\vec{v} \in C\left(\left[0, T_{\mu}\right]: \mathbb{H}^{s}\right) \cap C^{1}\left(\left[0, T_{\mu}\right]: \mathbb{H}^{s-2}\right)
$$

be another solution of (3.1), then the function $\vec{v}$ satisfies

$$
\partial_{t} \vec{v}(t)+A_{\mu} \vec{v}(t)+F(\vec{v}(t))=0,0 \leq t \leq T_{\mu}
$$

in $\mathbb{H}^{\mathbf{s}-2}(\mathbb{R})$. Then applying $\vec{W}_{\mu}(t-\tau)$ to $(4.2)$ for $0 \leq t \leq T_{\mu}$, we obtain

$$
\partial_{\tau} \vec{W}_{\mu}(t-\tau) \vec{v}(\tau)=\vec{W}_{\mu}(t-\tau) \partial_{\tau} \vec{v}(\tau)+\vec{W}_{\mu}(t-\tau) A_{\mu} \vec{v}(\tau)=-\vec{W}_{\mu}(t-\tau) F(\vec{v}(\tau)) .
$$

Integrating from 0 to $t$ and considering that $\vec{v}(0)=\vec{\phi}$, we have

$$
\vec{v}(t)=\vec{W}_{\mu}(t) \Phi-\int_{0}^{t} \vec{W}_{\mu}(t-\tau) F(\vec{v}(\tau)) d \tau
$$

in $\mathbb{H}^{\mathbf{s}-\mathbf{2}}$. Then from the theorem 2.2 we obtain as in the proof of the theorem 3.3 , since the second member of (4.3) is in $\mathbb{H}^{s}$. Therefore

$$
\vec{v} \in C\left(\left[0, T_{\mu}\right]: \mathbb{H}^{s}\right) \cap C^{1}\left(\left[0, T_{\mu}\right]: \mathbb{H}^{s-2}\right)
$$

is solution of the integral equation (3.3). Then the uniqueness established in theorem 3.2 means that $\vec{v}=\vec{u}_{\mu}$ in $\left[0, T_{\mu}\right]$ completing the proof of uniqueness.

The last statement follows immediately from the theorem 3.3.

We will then establish the existence of an interval $[0, T]$ independent of $\mu$, where all solutions $\vec{u}_{\mu}$ can be defined. This will be an essential result to show the continuous dependence on the initial data of the local solution of the problem of initial value (3.1).

Theorem 4.2. Let $\alpha, \beta \in C(\mathbb{R}) \cap L^{\infty}(\mathbb{R}), \mu>0, \Phi \in \mathbb{H}^{s}$ be given with $s>\frac{3}{2}$ and $\vec{u}_{\mu}$ the solution of the initial value problem (3.1) obtained in the theorem 4.1. Then, exists $T=T\left(\|\vec{\phi}\|_{\mathbb{H} s}, \alpha, \beta, s\right)>0$ such that $\vec{u}_{\mu}$ can be extended to the interval $[0, T]$. Moreover, there exists $\rho \in C([0, T], \mathbb{R})$ such that

$$
\left\{\begin{array}{l}
\left\|\vec{u}_{\mu}(t)\right\|_{\mathbb{H}^{s}}^{2} \leq \rho(t), 0 \leq t \leq T \\
\sup _{[0, T]} \rho(t) \leq C\left(\|\vec{\phi}\|_{\mathbb{H}^{s}}, \alpha, \beta, s, T\right)
\end{array}\right.
$$

where $\rho$ satisfies

$$
\left\{\begin{array}{l}
\rho^{\prime}(t)=2 C_{s} \lambda(t) \rho^{\frac{3}{2}}(t), t>0 \\
\rho(0)=\|\vec{\phi}\|_{\mathbb{H}^{s}}^{2}
\end{array}\right.
$$

Also, if $\vec{\phi} \in \mathbb{H}^{s+r}$ for $r \geq 0$, then for each $\mu>0$ we have

$$
\sup _{[0, T]}\left\|\vec{u}_{\mu}(t)\right\|_{\mathbb{H}^{s+r}}^{2} \leq C\left(\|\vec{\phi}\|_{\mathbb{H}^{s}}, \alpha, \beta, s, T\right)
$$


where $C(\cdot, \cdot, \cdot, \cdot, \cdot$,$) is increasing in each of its arguments.$

Proof. Let $\vec{u}_{\mu} \in C\left(\left[0, T_{\mu}\right]: \mathbb{H}^{s}\right) \cap C^{1}\left(\left[0, T_{\mu}\right]:, \mathbb{H}^{s-2}\right)$ be the solution of (3.1) given by the theorem 4.1. We have

$$
\partial_{t}\left\|\vec{u}_{\mu}\right\|_{\mathbb{H}^{s}}^{2}=2\left\langle\vec{u}_{\mu}, \partial_{t} \vec{u}_{\mu}\right\rangle_{\mathbb{H}^{s}}=2\left\langle\vec{u}_{\mu},-A_{\mu} \vec{u}_{\mu}\right\rangle_{\mathbb{H}^{s}}+2\left\langle\vec{u}_{\mu},-F\left(\vec{u}_{\mu}\right)\right\rangle_{\mathbb{H}^{s}} .
$$

From proposition 2.1 and proposition 3.1, we know that

$$
\left\langle\vec{u}_{\mu},-A_{\mu} \vec{u}_{\mu}\right\rangle_{\mathbb{H}^{s}} \leq 0
$$

hence

$$
\partial_{t}\left\|\vec{u}_{\mu}\right\|_{\mathbb{H}^{s}}^{2} \leq 2\left\langle\vec{u}_{\mu},-F\left(\vec{u}_{\mu}\right)\right\rangle_{\mathbb{H}^{s}} \leq 2\left|\left\langle\vec{u}_{\mu},-F\left(\vec{u}_{\mu}\right)\right\rangle_{\mathbb{H}^{s}}\right|=2\left|\left\langle\vec{u}_{\mu}, F\left(\vec{u}_{\mu}\right)\right\rangle_{\mathbb{H}^{s}}\right| .
$$

If $\vec{u}_{\mu}=(u, v)$, then by (3.2) we have

$$
\begin{aligned}
\left\langle\vec{u}_{\mu}, F\left(\vec{u}_{\mu}\right)\right\rangle_{\mathbb{H}^{s}}= & \alpha(t)\left\langle u, u \partial_{x} u\right\rangle_{H^{s}}+\left\langle u, v \partial_{x} v\right\rangle_{H^{s}}+\left\langle u, \partial_{x}(u v)\right\rangle_{H^{s}} \\
& +\left\langle v, u \partial_{x} u\right\rangle_{H^{s}}+\beta(t)\left\langle v, v \partial_{x} v\right\rangle_{H^{s}}+\left\langle v, \partial_{x}(u v)\right\rangle_{H^{s}} .
\end{aligned}
$$

By definition $\langle\cdot, \cdot\rangle_{H^{s}}=\left\langle J^{s} \cdot, J^{s} \cdot\right\rangle_{L^{2}}$ and equality $\left[J^{s}, f\right] g=J^{s}(f g)-f J^{s} g$, we have

$$
\left\langle u, v \partial_{x} v\right\rangle_{H^{s}}=\left\langle J^{s} u, J^{s}\left(v \partial_{x} v\right)\right\rangle_{L^{2}}=\left\langle J^{s} u,\left[J^{s}, v\right] \partial_{x} v\right\rangle_{L^{2}}+\left\langle J^{s} u, v J^{s} \partial_{x} v\right\rangle_{L^{2}},
$$

$\left\langle u, \partial_{x}(u v)\right\rangle_{H^{s}}=\left\langle J^{s} u,\left[J^{s}, u\right] \partial_{x} v\right\rangle_{L^{2}}+\left\langle J^{s} u, u J^{s} \partial_{x} v\right\rangle_{L^{2}}+\left\langle J^{s} u,\left[J^{s}, v\right] \partial_{x} u\right\rangle_{L^{2}}+\left\langle J^{s} u, v J^{s} \partial_{x} u\right\rangle_{L^{2}}$,

$$
\left\langle v, u \partial_{x} u\right\rangle_{H^{s}}=\left\langle J^{s} v,\left[J^{s}, u\right] \partial_{x} u\right\rangle_{L^{2}}+\left\langle J^{s} v, u J^{s} \partial_{x} u\right\rangle_{L^{2}},
$$

and

$$
\left\langle v, \partial_{x}(u v)\right\rangle_{H^{s}}=\left\langle J^{s} v,\left[J^{s}, u\right] \partial_{x} v\right\rangle_{L^{2}}+\left\langle J^{s} v, u J^{s} \partial_{x} v\right\rangle_{L^{2}}+\left\langle J^{s} v,\left[J^{s}, v\right] \partial_{x} u\right\rangle_{L^{2}}+\left\langle J^{s} v, v J^{s} \partial_{x} u\right\rangle_{L^{2}} .
$$

Furthermore, in the sum of (4.9), (4.10), (4.11) and (4.12), associating factors in the inner product $\langle\cdot, \cdot\rangle_{L^{2}}$, using integration by parts and the commutativity of the derivative with the potential of Bessel we have,

$$
\begin{gathered}
\left\langle J^{s} u, v J^{s} \partial_{x} v\right\rangle_{L^{2}}+\left\langle J^{s} v, v J^{s} \partial_{x} u\right\rangle_{L^{2}}+\left\langle J^{s} u, u J^{s} \partial_{x} v\right\rangle_{L^{2}}+\left\langle J^{s} v, u J^{s} \partial_{x} u\right\rangle_{L^{2}} \\
=-\left\langle\partial_{x}\left(v J^{s} u\right), J^{s} v\right\rangle_{L^{2}}+\left\langle J^{s} v, v \partial_{x} J^{s} u\right\rangle_{L^{2}}+\left\langle J^{s} u, u J^{s} \partial_{x} v\right\rangle_{L^{2}}-\left\langle\partial_{x}\left(u J^{s} v\right), J^{s} u\right\rangle_{L^{2}} \\
=-\left\langle\partial_{x} v \cdot J^{s} u, J^{s} v\right\rangle_{L^{2}}-\left\langle v \partial_{x} J^{s} u, J^{s} v\right\rangle_{L^{2}}+\left\langle J^{s} v, v \partial_{x} J^{s} u\right\rangle_{L^{2}} \\
\quad+\left\langle J^{s} u, u J^{s} \partial_{x} v\right\rangle_{L^{2}}-\left\langle u J^{s} \partial_{x} v, J^{s} u\right\rangle_{L^{2}}-\left\langle\partial_{x} u \cdot J^{s} v, J^{s} u\right\rangle_{L^{2}} \\
=-\left\langle\partial_{x} v \cdot J^{s} u, J^{s} v\right\rangle_{L^{2}}-\left\langle\partial_{x} u \cdot J^{s} v, J^{s} u\right\rangle_{L^{2}}
\end{gathered}
$$

Thus, replacing in (4.8), taking the absolute value and applying the triangular inequality, we get that

$$
\begin{aligned}
\left|\left\langle\vec{u}_{\mu}, F\left(\vec{u}_{\mu}\right)\right\rangle_{\mathbb{H}^{s}}\right| \leq & |\alpha(t)|\left|\left\langle u, u \partial_{x} u\right\rangle_{H^{s}}\right|+\left|\left\langle J^{s} u,\left[J^{s}, v\right] \partial_{x} v\right\rangle_{L^{2}}\right|+\left|\left\langle J^{s} u,\left[J^{s}, u\right] \partial_{x} v\right\rangle_{L^{2}}\right| \\
& +\left|\left\langle J^{s} u,\left[J^{s}, v\right] \partial_{x} u\right\rangle_{L^{2}}\right|+\left|\left\langle J^{s} u, v J^{s} \partial_{x} u\right\rangle_{L^{2}}\right|+\left|\left\langle J^{s} v,\left[J^{s}, u\right] \partial_{x} u\right\rangle_{L^{2}}\right| \\
& +|\beta(t)|\left|\left\langle v, v \partial_{x} v\right\rangle_{H^{s}}\right|+\left|\left\langle J^{s} v,\left[J^{s}, u\right] \partial_{x} v\right\rangle_{L^{2}}\right|+\left|\left\langle J^{s} v, u J^{s} \partial_{x} v\right\rangle_{L^{2}}\right| \\
& +\left|\left\langle J^{s} v,\left[J^{s}, v\right] \partial_{x} u\right\rangle_{L^{2}}\right|+\left|\left\langle\partial_{x} v \cdot J^{s} u, J^{s} v\right\rangle_{L^{2}}\right|+\left|\left\langle\partial_{x} u \cdot J^{s} v, J^{s} u\right\rangle_{L^{2}}\right| .
\end{aligned}
$$

Next we will estimate the second member of (4.14). Using the inequality (1.3) of the proposition 1.1 with $t=s$ and the regularity of the solution $\vec{u}_{\mu}$, we have

$$
\left|\left\langle u, u \partial_{x} u\right\rangle_{H^{s}}\right| \leq C_{s}\left\|\partial_{x} u\right\|_{H^{s-1}}\|u\|_{H^{s}}^{2} \leq C_{s}\|u\|_{H^{s}}^{3} \leq C_{s}\left(\|u\|_{H^{s}}^{2}+\|v\|_{H^{s}}^{2}\right)^{\frac{3}{2}}=C_{s}\left\|\vec{u}_{\mu}\right\|_{\mathbb{H}^{s}}^{3} .
$$

Combining the Cauchy-Schwartz inequality, the commutation estimate of the proposition 1.2 , the regularity of the solution $\vec{u}_{\mu}$ and the Sobolev immersion theorem, we obtain

$$
\begin{aligned}
\left|\left\langle J^{s} u,\left[J^{s}, v\right] \partial_{x} v\right\rangle_{L^{2}}\right| & \leq\left\|J^{s} u\right\|_{L^{2}}\left\|\left[J^{s}, v\right] \partial_{x} v\right\|_{L^{2}} \\
& \leq C_{s}\|u\|_{H^{s}}\left(\left\|\partial_{x} v\right\|_{L^{\infty}}\left\|J^{s-1} \partial_{x} v\right\|_{L^{2}}+\left\|J^{s} v\right\|_{L^{2}}\left\|\partial_{x} v\right\|_{L^{\infty}}\right) \\
& \leq C_{s}\|u\|_{H^{s}}\left(\left\|\partial_{x} v\right\|_{H^{s-1}}\left\|\partial_{x} v\right\|_{H^{s-1}}+\|v\|_{H^{s}}\left\|\partial_{x} v\right\|_{H^{s-1}}\right) \\
& \leq C_{s}\|u\|_{H^{s}}\|v\|_{H^{s}}^{2} \\
& \leq C_{s}\left\|\vec{u}_{\mu}\right\|_{\mathbb{H}^{s}}^{3}
\end{aligned}
$$




$$
\begin{aligned}
\left|\left\langle J^{s} u,\left[J^{s}, u\right] \partial_{x} v\right\rangle_{L^{2}}\right| & \leq\left\|J^{s} u\right\|_{L^{2}}\left\|\left[J^{s}, u\right] \partial_{x} v\right\|_{L^{2}} \\
& \leq C_{s}\|u\|_{H^{s}}\left(\|u\|_{H^{s}}\|v\|_{H^{s}}+\|u\|_{H^{s}}\|v\|_{H^{s}}\right) \\
& \leq C_{s}\left\|\vec{u}_{\mu}\right\|_{\mathbb{H}^{s}}^{3}
\end{aligned}
$$

and

$$
\left|\left\langle J^{s} u,\left[J^{s}, v\right] \partial_{x} u\right\rangle_{L^{2}}\right| \leq\left\|J^{s} u\right\|_{L^{2}}\left\|\left[J^{s}, v\right] \partial_{x} u\right\|_{L^{2}} \leq C_{s}\|u\|_{H^{s}}^{2}\|v\|_{H^{s}} \leq C_{s}\left\|\vec{u}_{\mu}\right\|_{\mathbb{H}^{s}}^{3} .
$$

In addition, from the Cauchy-Schwarz inequality, the regularity of the solution $\vec{u}_{\mu}$, integration by parts and the Sobolev immersion theorem, we have

$$
\left|\left\langle J^{s} u, v J^{s} \partial_{x} u\right\rangle_{L^{2}}\right|=\frac{1}{2}\left|\left\langle\partial_{x} v,\left(J^{s} u\right)^{2}\right\rangle_{L^{2}}\right| \leq C_{s}\|v\|_{H^{s}}\left\|J^{s} u\right\|_{L^{2}}^{2} \leq C_{s}\left\|\vec{u}_{\mu}\right\|_{\mathbb{H}^{s}}^{3} .
$$

and

$$
\begin{aligned}
\left|\left\langle\partial_{x} v \cdot J^{s} u, J^{s} v\right\rangle_{L^{2}}\right| & \leq\left\|\partial_{x} v\right\|_{L^{\infty}}\left\langle J^{s} u, J^{s} v\right\rangle_{L^{2}} \leq C_{s}\|v\|_{H^{s}}\left\|J^{s} u\right\|_{L^{2}}\left\|J^{s} v\right\|_{L^{2}} \\
& =C_{s}\|v\|_{H^{s}}\|u\|_{H^{s}}\|v\|_{H^{s}} \leq C_{s}\left\|\vec{u}_{\mu}\right\|_{\mathbb{H}^{s}}^{3}
\end{aligned}
$$

Similarly,

$$
\begin{cases}\left|\left\langle v, v \partial_{x} v\right\rangle_{H^{s}}\right| \leq C_{s}\left\|\vec{u}_{\mu}\right\|_{\mathbb{H}^{s}}^{3} & , \quad\left|\left\langle J^{s} v,\left[J^{s}, u\right] \partial_{x} u\right\rangle_{L^{2}}\right| \leq C_{s}\left\|\vec{u}_{\mu}\right\|_{\mathbb{H}^{s}}^{3}, \\ \left|\left\langle J^{s} v,\left[J^{s}, v\right] \partial_{x} u\right\rangle_{L^{2}}\right| \leq C_{s}\left\|\vec{u}_{\mu}\right\|_{\mathbb{H}^{s}}^{3} & , \quad\left|\left\langle J^{s} v,\left[J^{s}, u\right] \partial_{x} v\right\rangle_{L^{2}}\right| \leq C_{s}\left\|\vec{u}_{\mu}\right\|_{\mathbb{H}^{s}}^{3}, \\ \left|\left\langle\partial_{x} u \cdot J^{s} v, J^{s} u\right\rangle_{L^{2}}\right| \leq C_{s}\left\|\vec{u}_{\mu}\right\|_{\mathbb{H}^{s}}^{3}, & \left|\left\langle J^{s} v, u J^{s} \partial_{x} v\right\rangle_{L^{2}}\right| \leq C_{s}\left\|\vec{u}_{\mu}\right\|_{\mathbb{H}^{s}}^{3} .\end{cases}
$$

Therefore, replacing (4.15), (4.16), (4.17), (4.18), (4.19), (4.20) and (4.21), in (4.14) we obtain

$$
\left|\left\langle\vec{u}_{\mu}, F\left(\vec{u}_{\mu}\right)\right\rangle_{\mathbb{H}^{s}}\right| \leq C_{s}(1+|\alpha(t)|+|\beta(t)|)\left\|\vec{u}_{\mu}\right\|_{\mathbb{H}^{s}}^{3} \leq C_{s} \lambda(t)\left\|\vec{u}_{\mu}\right\|_{\mathbb{H}^{s}}^{3},
$$

where $\lambda(t)=1+|\alpha(t)|+|\beta(t)|>0$. After (4.7) and (4.22) we get

$$
\partial_{t}\left\|\vec{u}_{\mu}\right\|_{\mathbb{H}^{s}}^{2} \leq 2 C_{s} \lambda(t)\left\|\vec{u}_{\mu}\right\|_{\mathbb{H}^{s}}^{3}=2 \zeta\left(\left\|\vec{u}_{\mu}\right\|_{\mathbb{H}^{s}}^{2}\right)
$$

where $\zeta(y)=C_{s} \lambda(t) y^{\frac{3}{2}}$, for $y \geq 0$.

We consider $\rho^{\frac{1}{2}}(t)=\frac{\|\vec{\phi}\|_{\mathbb{H}^{s}}}{1-C_{s}\|\vec{\phi}\|_{\mathbb{H}^{s}} \int_{0}^{t} \lambda(\tau) d \tau}$, defined in the interval $\left[0, T^{*}\left[\right.\right.$ with $T^{*}=\frac{1}{C_{s}\|\vec{\phi}\|_{\mathbb{H}^{s}} \int_{0}^{t} \lambda(\tau) d \tau}$, the maximal solution of the problem of inicial value

$$
\left\{\begin{array}{l}
\rho^{\prime}(t)=2 \zeta(\rho(t)), t>0 \\
\rho(0)=\|\vec{\phi}\|_{s}^{2}
\end{array}\right.
$$

Then from (4.23), (4.24) and from the theory of ordinary differential equations, we have,

$$
\left\|\vec{u}_{\mu}\right\|_{\mathbb{H}^{s}}^{2} \leq \rho(t), t \in\left[0, T^{*}\left[\cap\left[0, T_{\mu}\right] .\right.\right.
$$

Thus for $\mu>0, \vec{u}_{\mu}$ it can be extended (if necessary) to an interval $[0, T]$. Furthermore, since $\lambda(\tau)$ is positive and continuous, we have

$$
1-C_{s}\|\vec{\phi}\|_{\mathbb{H}^{s}} \int_{0}^{t} \lambda(\tau) d \tau \geq 1-C_{s}\|\vec{\phi}\|_{\mathbb{H}^{s}} T\|\lambda\|_{L^{\infty}[0, T]}>0
$$

then

$$
\rho^{\frac{1}{2}}(t)=\frac{\|\vec{\phi}\|_{\mathbb{H}^{s}}}{1-C_{s}\|\vec{\phi}\|_{\mathbb{H}^{s}} \int_{0}^{t} \lambda(\tau) d \tau} \leq \frac{\|\vec{\phi}\|_{\mathbb{H}^{s}}}{1-C_{s}\|\vec{\phi}\|_{\mathbb{H}^{s}} T\|\lambda\|_{L^{\infty}[0, T]}}=C^{\frac{1}{2}}\left(\|\vec{\phi}\|_{\mathbb{H}^{s}}, \alpha, \beta, s, T\right) .
$$

From where we get that

$$
\sup _{[0, T]} \rho(t) \leq C\left(\|\vec{\phi}\|_{\mathbb{H}^{s}}, \alpha, \beta, s, T\right)
$$

This proves (4.4) as we wanted. Inequality (4.6) is obtained with the same arguments as above by writing $s+r$ instead of $s$. 
5. Continuous dependence on the initial data of the local solution. We conclude by showing in this section that $\vec{u}_{\mu}$ is continuously dependent on $\vec{\phi} \in \mathbb{H}^{s}, s>\frac{3}{2}$, in the sense that the application $\vec{\phi} \in \mathbb{H}^{s} \longmapsto \vec{u}_{\mu} \in$ $C\left([0, T]: \mathbb{H}^{s}\right)$ is continuous.

Theorem 5.1. Let $\alpha, \beta \in C(\mathbb{R}) \cap L^{\infty}(\mathbb{R}), \mu>0, \vec{\phi} \in \mathbb{H}^{s}$ be given with $s>\frac{3}{2}$ and $\vec{u}_{\mu, n} \in C\left([0, T]: \mathbb{H}^{s}\right)$ the solution of the initial value problem (3.1) satisfying (4.4). If $\left\{\vec{\phi}_{n}\right\}_{n \geq 1}$ is a sequence in $\mathbb{H}^{s}$ convergent to $\vec{\phi}$ in $\mathbb{H}^{s}$ and $\left\{\vec{u}_{\mu, n}\right\}_{n \geq 1}$ is a sequence of solutions from $(3.1)$ in $C\left(\left[0, T_{n}\right]: \mathbb{H}^{s}\right)$ with $\vec{u}_{\mu, n}(0)=\vec{\phi}_{n}$, then for all $\bar{T} \in] 0, T\left[\right.$ satisfies that for $n$ enough large, $\vec{u}_{\mu, n}$ is defined in $[0, \bar{T}]$ and

$$
\lim _{n \rightarrow+\infty} \sup _{[0, \bar{T}]}\left\|\vec{u}_{\mu, n}(t)-\vec{u}_{\mu, n}(t)\right\|_{\mathbb{H}^{s}}=0
$$

Proof. From the theorem 4.2, for all $n$ we have

$$
\left\|\vec{u}_{\mu, n}(t)\right\|_{\mathbb{H}^{s}}^{2} \leq \rho_{n}(t) \quad t \in\left[0, T_{n}\right] .
$$

where $\rho_{n}$ satisfies

$$
\left\{\begin{array}{l}
\rho_{n}^{\prime}(t)=2 C_{s} \lambda(t) \rho_{n}^{\frac{3}{2}}(t), t>0 \\
\rho_{n}(0)=\left\|\vec{\phi}_{n}\right\|_{\mathbb{H}^{s}}^{2},
\end{array}\right.
$$

in $\left[0, T_{n}^{*}\left[\right.\right.$ with $T_{n}^{*}=\frac{1}{C_{s}\|\vec{\phi}\|_{\mathbb{H}^{s}} \int_{0}^{t} \lambda(\tau) d \tau}$ and $\left.T_{n} \in\right] 0, T_{n}^{*}[$. Now, for $\bar{T} \in] 0, T\left[\right.$ consider $\varepsilon=\varepsilon\left(\bar{T},\|\vec{\phi}\|_{\mathbb{H}^{s}}\right)>0$ such that

$$
\varepsilon+\|\vec{\phi}\|_{\mathbb{H}^{s}} \leq\left(\frac{T}{\bar{T}}\right)\|\vec{\phi}\|_{\mathbb{H}^{s}},
$$

then, exists $N_{0}=N(\varepsilon)$ such that $\left\|\vec{\phi}_{n}-\vec{\phi}\right\|_{\mathbb{H}^{s}}<\varepsilon$ for $n \geq N_{0}$. Then from the definition of $\rho_{n}$ we have for $n \geq N_{0}$,

$$
\rho_{n}^{\frac{1}{2}}(t)=\frac{\left\|\vec{\phi}_{n}\right\|_{\mathbb{H}^{s}}}{1-C_{s}\left\|\vec{\phi}_{n}\right\|_{\mathbb{H}^{s}} \int_{0}^{t} \lambda(\tau) d \tau} \leq \frac{\varepsilon+\|\vec{\phi}\|_{\mathbb{H}^{s}}}{1-C_{s}\left(\varepsilon+\left\|\vec{\phi}_{n}\right\|_{\mathbb{H}^{s}}\right) \int_{0}^{t} \lambda(\tau) d \tau} \equiv \rho_{\bar{T}}(t),
$$

for $t \in[0, \bar{T}]$, since of $(5.2)$ we have

$$
C_{s} t \int_{0}^{t} \lambda(\tau) d \tau\left(\varepsilon+\left\|\vec{\phi}_{n}\right\|_{\mathbb{H}^{s}}\right) \leq C_{s}\left(\varepsilon+\left\|\vec{\phi}_{n}\right\|_{\mathbb{H}^{s}}\right) \bar{T} \int_{0}^{t} \lambda(\tau) d \tau \leq C_{s}\left\|\vec{\phi}_{n}\right\|_{\mathbb{H}^{s}} T \int_{0}^{t} \lambda(\tau) d \tau<1,
$$

where the last inequality follows from the choice of $T$. So we obtain

$$
\sup _{[0, \bar{T}]} \rho_{n}(t) \leq C\left(\|\vec{\phi}\|_{\mathbb{H}^{s}}, \alpha, \beta, s, \bar{T},\right) .
$$

Thus, $\vec{u}_{\mu, n}(t)$ can be extended to $[0, \bar{T}]$, satisfying $(5.1)$.

Now we consider $\vec{w}=\left(w_{1}, w_{2}\right)=\left(u-u_{n}, v-v_{n}\right)=\vec{u}_{\mu, n}-\vec{u}_{\mu, n}$, for the components of $\vec{w}$ we have

$$
\begin{aligned}
\partial_{t} w_{1}= & -\partial_{x}^{3} w_{1}-\frac{\alpha(t)}{p+1} \partial_{x}\left(u^{p+1}-u_{n}^{p+1}\right)-\frac{1}{p+1} \partial_{x}\left(v^{p+1}-v_{n}^{p+1}\right)-\partial_{x}\left(u v^{p}-u_{n} v_{n}^{p}\right)+\mu \partial_{x}^{2} w_{1} \\
= & -\partial_{x}^{3} w_{1}-\frac{1}{p+1} \partial_{x}\left(\alpha(t) w_{1} \sum_{j=1}^{p+1} u^{p+1-j} u_{n}^{j-1}+w_{2} \sum_{j=1}^{p+1} v^{p+1-j} v_{n}^{j-1}\right) \\
& -\partial_{x}\left(u w_{2} \sum_{j=1}^{p} v^{p-j} v_{n}^{j-1}+w_{1} v_{n}^{p}\right)+\mu \partial_{x}^{2} w_{1} .
\end{aligned}
$$

Similarly

$$
\begin{aligned}
\partial_{t} w_{2}= & -\partial_{x}^{3} w_{2}-\frac{1}{p+1} \partial_{x}\left(w_{1} \sum_{j=1}^{p+1} u^{p+1-j} u_{n}^{j-1}+\beta(t) w_{2} \sum_{j=1}^{p+1} v^{p+1-j} v_{n}^{j-1}\right) \\
& -\partial_{x}\left(v w_{1} \sum_{j=1}^{p} u^{p-j} u_{n}^{j-1}+w_{2} u_{n}^{p}\right)+\mu \partial_{x}^{2} w_{2} .
\end{aligned}
$$



have

Then, using definition of the inner product in $\mathbb{H}^{r}$, the equality $\left\langle u, \partial_{x}^{3} u\right\rangle_{H^{s}}=0$ and integration by parts, we

$$
\begin{aligned}
\frac{1}{2} \partial_{t}\|\vec{w}(t)\|_{\mathbb{H}^{s}}^{2}= & \left\langle w_{1}, \partial_{t} w_{1}\right\rangle_{H^{s}}+\left\langle w_{2}, \partial_{t} w_{2}\right\rangle_{H^{s}} \\
= & \frac{1}{p+1}\left\langle\partial_{x} w_{1}, \alpha(t) w_{1} \sum_{j=1}^{p+1} u^{p+1-j} u_{n}^{j-1}+w_{2} \sum_{j=1}^{p+1} v^{p+1-j} v_{n}^{j-1}\right\rangle \\
& +\left\langle\partial_{x} w_{1}, u w_{2} \sum_{j=1}^{p} v^{p-j} v_{n}^{j-1}+w_{1} v_{n}^{p}\right\rangle_{H^{s}}-\mu\left\|\partial_{x} w_{1}\right\|_{H^{s}}^{2} \\
& +\frac{1}{p+1}\left\langle\partial_{x} w_{2}, w_{1} \sum_{j=1}^{p+1} u^{p+1-j} u_{n}^{j-1}+\beta(t) w_{2} \sum_{j=1}^{p+1} v^{p+1-j} v_{n}^{j-1}\right\rangle_{H^{s}} \\
& +\left\langle\partial_{x} w_{2}, v w_{1} \sum_{j=1}^{p} u^{p-j} u_{n}^{j-1}+w_{2} u_{n}^{p}\right\rangle_{H^{s}}-\mu\left\|\partial_{x} w_{2}\right\|_{H^{s}}^{2} .
\end{aligned}
$$

Using the proposition 4.1, the Cauchy-Schwartz inequality and the fact that $H^{s}$ is a Banach algebra if $s>\frac{1}{2}$, we obtain from (5.4) and (5.5)

$$
\begin{aligned}
\frac{1}{2} \partial_{t}\|\vec{w}(t)\|_{\mathbb{H}^{s}}^{2} \leq & C\left\|\partial_{x} \vec{w}\right\|_{H^{s}}\left\|w_{1} \sum_{j=1}^{p+1} u^{p+1-j} u_{n}^{j-1}+w_{2} \sum_{j=1}^{p+1} v^{p+1-j} v_{n}^{j-1}\right\|_{H^{s}} \\
& +C\left\|\partial_{x} \vec{w}\right\|_{H^{s}}\left\|u w_{2} \sum_{j=1}^{p} v^{p-j} v_{n}^{j-1}+w_{1} v_{n}^{p}\right\|_{H^{s}} \\
& +C\left\|\partial_{x} \vec{w}\right\|_{H^{s}}\left\|v w_{1} \sum_{j=1}^{p} u^{p-j} u_{n}^{j-1}+w_{2} u_{n}^{p}\right\|_{H^{s}}-\mu\left\|\partial_{x} \vec{w}\right\|_{\mathbb{H}^{s}}^{2}
\end{aligned}
$$

By the inequalities (4.4), (5.1) and (5.3) we obtain

$$
\partial_{t}\|\vec{w}(t)\|_{\mathbb{H}^{s}}^{2} \leq C\|\vec{w}\|_{\mathbb{H}^{s}}\left\|\partial_{x} \vec{w}\right\|_{\mathbb{H}^{s}}-2 \mu\left\|\partial_{x} \vec{w}\right\|_{\mathbb{H}^{s}}^{2} \leq \varepsilon\|\vec{w}\|_{\mathbb{H}^{s}}^{2}+\frac{C^{2}}{4 \varepsilon}\left\|\partial_{x} \vec{w}\right\|_{\mathbb{H}^{s}}^{2}-2 \mu\left\|\partial_{x} \vec{w}\right\|_{\mathbb{H}^{s}}^{2}
$$

where $C=C\left(\|\vec{\phi}\|_{\mathbb{H}^{s}}, \lambda, s, \bar{T}\right)$ and Cauchy's inequality with $\varepsilon$ has been used. Choosing $\varepsilon=\frac{C^{2}}{8 \mu}$ we obtain

$$
\partial_{t}\|\vec{w}(t)\|_{\mathbb{H}^{s}}^{2} \leq \frac{C^{2}}{8 \mu}\|\vec{w}(t)\|_{\mathbb{H}^{s}}^{2}
$$

Integrating from 0 to $t$, follows that

$$
\|\vec{w}(t)\|_{\mathbb{H}^{s}}^{2} \leq\|\vec{w}(0)\|_{\mathbb{H}^{s}}^{2}+\frac{C^{2}}{8 \mu} \int_{0}^{t}\|\vec{w}(\tau)\|_{\mathbb{H}^{s}}^{2} d \tau
$$

Applying Gronwall inequality we get

$$
\|\vec{w}(t)\|_{\mathbb{H}^{s}}^{2} \leq\|\vec{w}(0)\|_{\mathbb{H}^{s}}^{2}\left[1+\frac{C^{2}}{8 \mu} \bar{T} \exp \left(\frac{C^{2}}{8 \mu} \bar{T}\right)\right]=C_{\mu}\|\vec{w}(0)\|_{\mathbb{H}^{s}}^{2}
$$

which shows

$$
\sup _{[0, \bar{T}]}\left\|\vec{u}_{\mu, n}(t)-\vec{u}_{\mu}(t)\right\|_{\mathbb{H}^{s}}^{2} \leq C_{\mu}\left\|\vec{\phi}_{n}-\vec{\phi}\right\|_{\mathbb{H}^{s}}^{2}
$$

This conclude the proof.

\section{REFERENCES}

[1] Albert, J., Bona, J., Saut, J.C., Model equations for waves in stratified fluids. Proc. Royal Soc. London A, (1997) 453, pp. 1233-1260.

[2] Bona, J., Chen, H, Solitary waves in nonlinear dispersive systems. Discrete and continuous dynamical systems B, 2 (2002), pp. $313-378$. 
[3] Bona,J, Chen, H., Karakashian, o., Stability of solitary-wave solutions of systems of dispersive equations. Applied Mathematics \& Optimization., Volume 75, Issue 1, (2017), pp. 27-53.

[4] Bona, J., Chen, M., Saut, J.C. Boussinesq equations and other systems for small-amplitude long waves in nonlinear dispersive media. I. Derivation and linear theory. J. Nonlinear Sci. 12 (2002), pp. 283-318.

[5] - Boussinesq equations and other systems for small-amplitude long waves in nonlinear dispersive media. II. The nonlinear theory. Nonlinearity 17 (2004), pp. 925-952.

[6] Bona, J., Cohen,J., Wang, G., Global well-posedness for a system of KdV-type equations with coupled quadratic nonlinearities. Nagoya Math. J. Volume 215 (2014), pp. 67-149.

[7] Gear, J. A., Grimshaw, R., Weak and strong interactions between internal solitary waves. Stud. Appl. Math., 70, (1984), pp. $235-258$.

[8] Hu, H., Liu, Q.P., Decouple a coupled KdV system of Nutku and Og̃uz. Phys. Lett. 294A (2002), pp. 84-86.

[9] Iório Jr. R.J., On the Cauchy problem for the Benjamin-Ono equation. Comm. PDE, 11, (1986), pp. 1031-1081.

[10] - KdV, BO and friends in weigheted Sobolev spaces. Springer-Verlag, Lecture Notes in Mathematics, 1450, (1990), pp. 104-121.

[11] Kato, T. On the Cauchy problem for the (Generalized) KdV equations. Studies in Applied Mathematics, Advances in Mathematics Supplementary Studies, 8, (1983), pp. 93-128.

[12] Kato, T., Fujita, H., On the non-stationary Navier-Stokes system. Red. Sem. Mat. Uni. Padova, 32, (1962), pp. $243-260$.

[13] Kato, T., Ponce, G., Commutator estimates and the Euler and Navier-Stokes equations. Comm. Pure Appl. Math. 41, (1988), pp. 891907.

[14] Majda, A., Biello, J. The nonlinear interaction of barotropic and equatorial baroclinic Rossby waves. J. Atmospheric Sci. 60 (2003), pp. 1809-1821.

[15] Montealegre, J., El sistema de Nutku-Õ̃uz I: Buena formulación global en espacios de alta regularidad. Por publicar.

[16] - El sistema de Nutku-Õguz II: Buena formulación global en espacios de baja regularidad. Por publicar.

[17] Montealegre, J., Monzón, C. Existencia y unicidad de solución local para un sistema dispersivo con coeficientes dependientes del tiempo. Reporte de investigación, $\mathrm{N}^{\circ} 20$ Serie B, PUCP, (2006).

[18] Nutku, Y., Og̃uz, Ö., Bi-Hamiltonian structure of a pair of coupled kdv equations. Il Nuovo Cimento 105B (1990), pp. 1381-1383. 